Article

\title{
Distinct and Overlapping Patterns of Acute Ethanol-Induced C-Fos Activation in Two Inbred Replicate Lines of Mice Selected for Drinking to High Blood Ethanol Concentrations
}

\author{
Stacey L. Robinson 1,2,+, Ana Paula S. Dornellas 1,2,+(D), Nathan W. Burnham ${ }^{3}$, \\ Christa A. Houck 1,2, Kendall L. Luhn ${ }^{1}$, Sophie C. Bendrath 1,2 (D), Michel A. Companion 1,2, \\ Honoreé W. Brewton ${ }^{1,2} \mathbb{D}$, Rhiannon D. Thomas ${ }^{1,2}$, Montserrat Navarro ${ }^{1,2}$ \\ and Todd E. Thiele ${ }^{1,2, *(D)}$ \\ 1 Department of Psychology \& Neuroscience, The University of North Carolina, Chapel Hill, NC 27599, USA; \\ slrobin@email.unc.edu (S.L.R.); lopera@email.unc.edu (A.P.S.D.); chouck@email.unc.edu (C.A.H.); \\ kluhn@email.unc.edu (K.L.L.); sophiecb@ad.unc.edu (S.C.B.); macompan@live.unc.edu (M.A.C.); \\ honoreeb@live.unc.edu (H.W.B.); thomasrd@unc.edu (R.D.T.); mthiele@email.unc.edu (M.N.) \\ 2 Bowles Center for Alcohol Studies, The University of North Carolina, Chapel Hill, NC 27599, USA \\ 3 Department of Chemistry, North Carolina State University, Raleigh, NC 27695, USA; nwburnha@ncsu.edu \\ * Correspondence: thiele@unc.edu; Tel.: +1-919-966-1519; Fax: +1-919-962-2537 \\ + These authors contributed equally to this work.
}

Received: 14 November 2020; Accepted: 10 December 2020; Published: 15 December 2020

check for updates

\begin{abstract}
The inbred high drinking in the dark (iHDID1 and iHDID2) strains are two replicate lines bred from the parent $\mathrm{HS} / \mathrm{Npt}$ (HS) line for achieving binge levels of blood ethanol concentration $(\geq 80 \mathrm{mg} / \mathrm{dL}$ BEC) in a four-hour period. In this work, we sought to evaluate differences in baseline and ethanol-induced c-Fos activation between the HS, iHDID1, and iHDID2 genetic lines in brain regions known to process the aversive properties of ethanol. Methods: Male and female HS, iHDID1, and iHDID2 mice underwent an IP saline $23 \mathrm{~g} / \mathrm{kg}$ ethanol injection. Brain sections were then stained for c-Fos expression in the basolateral/central amygdala (BLA/CeA), bed nucleus of the stria terminals (BNST), A2, locus coeruleus (LC), parabrachial nucleus (PBN), lateral/medial habenula $(\mathrm{LHb} / \mathrm{MHb})$, paraventricular nucleus of the thalamus (PVT), periaqueductal gray (PAG), Edinger-Westphal nuclei (EW), and rostromedial tegmental nucleus (RMTg). Results: The iHDID1 and iHDID2 lines showed similar and distinct patterns of regional c-Fos; however, in no region did the two both significantly differ from the HS line together. Conclusions: Our findings lend further support to the hypothesis the iHDID1 and the iHDID2 lines arrive at a similar behavior phenotype through divergent genetic mechanisms.
\end{abstract}

Keywords: HDID; c-fos; mice; ethanol; alcohol; drinking in the dark; taste aversion

\section{Introduction}

The predisposition to consume high amounts of ethanol in a short period of time, or 'binge-like' behavior, has been extensively demonstrated to serve as a predictive measure for the development of ethanol dependence and is further a dangerous behavior associated with numerous health risks [1,2]. While extensive work has demonstrated a genetic contribution to ethanol preference and predisposition to withdrawal from chronic ethanol use, factors underlying the drive to binge consume ethanol have received relatively less attention. To enable investigation into the genetic underpinnings of binge ethanol intake, the Crabbe research group developed the high drinking in the dark (HDID) strain, 
a line selected from an original heterogeneous breeding stock (HS/Npt or HS), for high blood ethanol concentration at the end of a four-hour binge period [3]. Following the creation of the HDID1 strain, a replicate breeding process was performed to create the HDID2 strain. To stabilize each genotype, the group then established an inbred version of each line (iHDID1 and iHDID2) which we utilize in this work [4]. While no rodent line can fully model the full spectrum of human alcohol use disorders, these lines present an opportunity to gain insights into genetically determined mechanisms which predispose individuals to achieve high blood ethanol concentrations in a short period of time.

Though generated using identical criteria, these two strains display significant differences in ethanol drinking pattern (microstructure) and brain gene coexpression networks relative to both the HS line and each other, suggesting polygenetic origins of the same behavioral end point $[5,6]$. The HDID1 line consumes ethanol in the same number of bouts as HS, but ingests larger amounts of ethanol during each individual bout [5]. This difference in bout size was not observed in water or saccharine consumption in this study, suggesting that this behavior is ethanol specific. This is notable as early 'gulping' of ethanol, or consumption of large amounts of ethanol in a single drinking bout, has been shown in non-human primates to be predictive of future chronic heavy drinking [7]. Further, larger bout sizes are associated with a relative increased 'liking' of ethanol which is thought to be driven by an increased experience of the positive or decreased experience of the aversive properties of ethanol $[8,9]$. This may suggest a pathway through which HDID1 animals are susceptible to heavy drinking, distinct from the higher number of bouts consumed by HDID2 animals [5]. Several studies have suggested that the HDID1 and iHDID1 lines may possess an intriguing resistance to the aversive properties of ethanol consumption. While HDID1 and iHDID1 mice develop a conditioned taste aversion (CTA) similar to that of HS mice following lithium chloride exposure, they display an attenuated CTA to $2 \mathrm{~g} / \mathrm{kg}$ (but not $4 \mathrm{~g} / \mathrm{kg}$ ) ethanol injection $[4,10]$. This may suggest that relatively higher levels of ethanol intoxication are required to generate the experience of the aversive properties of ethanol in this strain. That no difference between the HS and the original HDID1 and HDID2 genotypes in ethanol conditioned place preference (CPP) were observed in this work further suggests a reduction in the aversive effects of ethanol, rather than an enhancement of the reinforcing properties, may underlie increased consumption. A lack of change in the relative level of anxiolysis induced by ethanol consumption or injection compared to HS mice lends further support to this hypothesis [11].

The more recently generated HDID2 line appears to display less ethanol-specific alteration in behaviors. HDID2 animals tend to consume high levels of ethanol through a larger number of similarly sized bouts relative to HS or HDID1 animals for both ethanol and water, suggesting that they may show a generalized increase in intake [5]. They further display a more generalized reduction in CTA behavior, showing less CTA to both $2 \mathrm{~g} / \mathrm{kg}$ ethanol and lithium chloride exposure [10,12]. Similar to the iHDID1 line the iHDID2 strain possess a similar level of ethanol CPP and anxiolysis following ethanol exposure as HS animals, suggesting that this line may also have little relative change in processing the reinforcing effects of ethanol versus the parent HS line. Additionally, the iHDID2 line shows reduced sensitivity to the aversive effects of ethanol relative to the HS mice [4,11].

In the present study, we assessed acute ethanol-induced neural activation, as measure by c-Fos expression, in the HS, iHDID1 and iHDID2 lines. The guiding hypotheses of this study were that a) the iHDID lines would show significant differences in c-Fos activation relative to the HS line in brain regions modulating ethanol consumption (specifically those involved in the aversive properties of ethanol) leading to binge-like BECs and b), that the iHDID1 and HHDID2 lines would show significant differences in c-Fos activation in a subset of brain regions, which may provide insight into line differences in drinking microstructure and genetic networks outlined above. Given the attention dedicated in previous studies to the relative processing of the aversive properties of ethanol, this current work focused on c-Fos activation within multiple (though in no way inclusive of all) mid- and hindbrain regions associated with these properties. These doses were chosen based on previous work that assessed CTA sensitivity in the HS, iHDID1, and iHDID2 lines [4] The present results provide insight 
into the potentially divergent patterns of neural activity which underlie the same ultimate behavioral phenotype of binge-like drinking behavior in these two independently generated high drinking strains.

\section{Methods}

\subsection{Animals}

Inbred high drinking in the dark (iHDID1 (generation S26.F20), iHDID2 (generation S32.F10) and the founder HS/NPT (HS) mice (generation G95) were group housed 2-5 per cage from weaning until the beginning of experiments, with a room temperature maintained at $22{ }^{\circ} \mathrm{C}$ and a $12: 12 \mathrm{~h}$ forward light/dark cycle with lights on at 7:00 am. Ad libitum Prolab ${ }^{\circledR}$ RMH 3000 (Purina labDiet ${ }^{\circledR}$; St. Louis, MO, USA) chow and water were available. All protocols were conducted under National Institute of Health guidelines and were approved by the University of North Carolina Institutional Animal Care and Use Committee (protocols 20-165.0 and 18-096.0).

\subsection{Immunohistochemistry}

A total of 15 male and 15 female mice (6-8 weeks old) from each independent lines (iHDID1, iHDID2) and HS/NPT (HS) were used in each dose condition for a total of 90 mice. Due to tissue processing complications, not all brains were viable for analysis for every brain area. Individual Ns for each genotype, treatment, and sex are provided in the results for each region.

One week before the experiments, mice were habituated to single housing. On the test day, mice were intraperitoneally (IP) injected with ethanol (Decon Laboratories, code 2801, King of Prussia, PA, USA) at 2 or $3 \mathrm{~g} / \mathrm{kg}$ (made up as 20\% (v/v)) or saline (Hospira, code NDC 0409-4888-02, Lake Forest, IL, USA) (equivalent to the $3 \mathrm{~g} / \mathrm{kg}$ volume of liquid) two hours before perfusions. Mice were weighed and anesthetized with ketamine/xylazine (Ketaset, code EA2489-564, Kalamazoo, MI, USA; AnaSed, code NDC 59399-111-50, Lake Forest, IL, USA) overdose (100/10 mg/kg) and transcardially perfused with phosphate-buffered saline (PBS; Sodium phosphate monobasic, VWR Life Science, code 0571, Solon, OH, USA; Sodium phosphate dibasic, ACS, code 0404, Solon, OH, USA; Sodium chloride, Macron Fine Chemicals, code 7581-12, Radnor, PA, USA) for 1 min followed immediately by $10 \%$ formalin (Fisher Scientific, code SF100-20, Hampton, NH, USA) for 2 min using a Masterflex L/S perfusion pump (catalogue \#7200-12, Cole-Parmer, Vernon Hills, IL, USA) in a rate of 7-8 mL/min.

Brains were collected and postfixed in $10 \%$ formalin for $24 \mathrm{~h}$, and, next, were cryoprotected in 0.2 M Phosphate buffer (PB; Sodium phosphate monobasic, VWR Life Science, code 0571, Solon, OH, USA; Sodium phosphate dibasic, ACS, code 0404, Solon, OH, USA) at pH 7.4 containing 50\% ethylene glycol (Fisher, catalogue \#E178500, Hampton, NH, USA) and 0.01\% polyvinyl-pyrrolidone (VWR Life Science, catalogue \#EM-7220, Radnor, PA, USA). Brains were sectioned using a vibratome into $40 \mu \mathrm{m}$ slices (model VT1000 S, Leica Biosystems, Buffalo Grove, IL, USA). Tissue was placed in cryopreserve and stored in $\mathrm{a}-20^{\circ} \mathrm{C}$.

Sections underwent three rinses ( 2 min each) prior to overnight incubation in rabbit anti-c-Fos (catalogue \#226 003, Synaptic Systems, Goettingen, Germany), diluted 1:1000 in PBS with 0.25\% Triton-X (catalogue T9484, Sigma-Aldrich, St. Louis, MO, USA) and 0.01\% sodium azide (D5637, Sigma-Aldrich, St. Louis, MO, USA). Following two 2 min rinses, sections incubated for $30 \mathrm{~min}$ in biotinylated donkey anti-rabbit secondary (code \#711-065-152, Jackson Immunoresearch, West Grove, PA, USA) diluted 1:1000 in PBS. Sections underwent four additional rinses before incubating for $1 \mathrm{~h}$ in Vectastain ABC kit (Vector Laboratories, Burlingame, CA, USA) diluted 1:1000 in PBS. Sections rinsed twice before exposure to PBS containing $0.05 \%$ diaminobenzidine tetrahydrochloride hydrate (DAB; catalogue \#D5637, Sigma-Aldrich, St. Louis, MO, USA), $0.05 \%$ ammonium nickel (II) sulfate hexahydrate (catalogue \#A-1827, Sigma-Aldrich, St. Louis, MO, USA), and 0.01\% hydrogen peroxide (30\% in water) (catalogue \#BP2633500, Fisher Scientific, Fair Lawn, NJ, USA). The stain was finalized by rinses in PBS. 


\subsection{C-Fos Quantification}

Sections were imaged using a Nikon e400 biological microscope with a digital sight ds-u1 imaging attachment (Nikon Instruments Inc., Melville, NY, USA). Brain region coordinates were identified with use of The Mouse Brain in Stereotaxic Coordinates atlas [13]. Images were then analyzed using Icy bioimage informatics open source software version 1.0 (Institut Pasteur and France-BioImaging, Paris, France) [14]. Each c-Fos value represents 2-6 bilateral images per animal averaged together. Images were independently quantified by 2-3 investigators blind to genotype and drug treatment of each animal. The results generated by each investigator were then averaged together to produce the final value for each animal to insure lack of bias during image quantification.

\subsection{Statistics}

Analyses were performed in GraphPad Prism 8.0 (GraphPad Software, La Jolla, CA, USA). One-way analysis of variance (ANOVA) and Dunnett's post hoc test were used to assess relative c-Fos reactivity across genotypes in saline treated animals. A two-way ANOVA (genotype $\times$ dose) and Tukey's multiple comparisons test were used to assess relative c-Fos activation in each genotype following the 2 or $3 \mathrm{~g} / \mathrm{kg}$ ethanol injection. Significance was defined as $p<0.05$. Because of loss of tissue due to damage noted above, there was not sufficient sample size to detect sex effects. Given previous work on ethanol-induced CTA in these lines observed no sex-dependent differences and thus reported results of analyses collapsed on sex [4], we likewise collapsed data across sex in the present analyses.

\section{Results}

\subsection{C-Fos Response in the Hindbrain}

A2 numbers: HS saline, $n=8(\mathrm{~F}=4, \mathrm{M}=4), 2 \mathrm{~g} / \mathrm{kg}, n=10(\mathrm{~F}=5, \mathrm{M}=5), 3 \mathrm{~g} / \mathrm{kg}, n=8(\mathrm{~F}=5$, $\mathrm{M}=3)$; iHDID1 saline, $n=5(\mathrm{~F}=2, \mathrm{M}=3), 2 \mathrm{~g} / \mathrm{kg}, n=9(\mathrm{~F}=6, \mathrm{M}=3), 3 \mathrm{~g} / \mathrm{kg}, n=9(\mathrm{~F}=4, \mathrm{M}=5)$; iHDID2 saline, $n=9(\mathrm{~F}=5, \mathrm{M}=4), 2 \mathrm{~g} / \mathrm{kg}, n=9(\mathrm{~F}=4, \mathrm{M}=5), 3 \mathrm{~g} / \mathrm{kg}, n=9(\mathrm{~F}=4, \mathrm{M}=5)$. Locus coeruleus (LC) numbers: HS saline, $n=5(\mathrm{~F}=1, \mathrm{M}=4), 2 \mathrm{~g} / \mathrm{kg}, n=9(\mathrm{~F}=4, \mathrm{M}=5), 3 \mathrm{~g} / \mathrm{kg}, n=7$ $(\mathrm{F}=5, \mathrm{M}=2)$; iHDID1 saline, $n=7(\mathrm{~F}=3, \mathrm{M}=4), 2 \mathrm{~g} / \mathrm{kg}, n=9(\mathrm{~F}=5, \mathrm{M}=4), 3 \mathrm{~g} / \mathrm{kg}, n=8(\mathrm{~F}=3$, $\mathrm{M}=5)$; iHDID2 saline, $n=5(\mathrm{~F}=2, \mathrm{M}=3), 2 \mathrm{~g} / \mathrm{kg}, n=7(\mathrm{~F}=2, \mathrm{M}=5), 3 \mathrm{~g} / \mathrm{kg}, n=9(\mathrm{~F}=5, \mathrm{M}=4)$. Parabrachial nucleus (PBN) numbers: HS saline $n=9(\mathrm{~F}=4, \mathrm{M}=5), 2 \mathrm{~g} / \mathrm{kg}, n=8(\mathrm{~F}=4, \mathrm{M}=4), 3 \mathrm{~g} / \mathrm{kg}$, $n=5(\mathrm{~F}=3, \mathrm{M}=2) ;$ iHDID1 saline, $n=6(\mathrm{~F}=3, \mathrm{M}=3), 2 \mathrm{~g} / \mathrm{kg}, n=7(\mathrm{~F}=3, \mathrm{M}=4), 3 \mathrm{~g} / \mathrm{kg}, n=10(\mathrm{~F}=5$, $\mathrm{M}=5)$; iHDID2 saline, $n=7(\mathrm{~F}=3, \mathrm{M}=4), 2 \mathrm{~g} / \mathrm{kg}, n=8(\mathrm{~F}=3, \mathrm{M}=5), 3 \mathrm{~g} / \mathrm{kg}, n=11(\mathrm{~F}=6, \mathrm{M}=5)$.

C-Fos immunoreactivity following injection of 0 (saline) was assessed by one-way ANOVA and Dunnett's post hoc test in male and female HS, iHDID1, and iHDID2 mice to assess potential baseline differences in regional activity in response to injection. One-way ANOVA detected no significant differences between genotypes in the A2 $(\mathrm{F}(2,19)=3.466, p=0.0521), \mathrm{LC}(\mathrm{F}(2,14)=3.165, p=0.0734)$, $\operatorname{PBN}(\mathrm{F}(2,19)=1.248, p=0.3097)$ Figure $1 \mathrm{~A}-\mathrm{C}$.

The change in percent c-Fos expression relative to the saline injection was then assessed following a 2 or $3 \mathrm{~g} / \mathrm{kg}$ ethanol injection with two-way ANOVA (genotype $\times$ dose) and Tukey's multiple comparisons test. In the A2, a significant effect of genotype $(\mathrm{F}(2,67)=19.96, p<0.0001)$, dose $(\mathrm{F}(2,67)=53.47$, $p<0.0001)$ and an interaction of genotype $\times$ dose $(F(4,67)=7.093, p<0.0001)$ were detected. Post hoc analysis found a significant increase in c-Fos expression relative to saline injection in the HS ( $2 \mathrm{~g} / \mathrm{kg}$ ethanol: $p=0.0001 ; 3 \mathrm{~g} / \mathrm{kg}$ ethanol: $p<0.0001)$, iHDID1 $(2 \mathrm{~g} / \mathrm{kg}$ ethanol: $p=0.0209$; $3 \mathrm{~g} / \mathrm{kg}$ ethanol: $p=0.0589)$, and iHDID2 $(2 \mathrm{~g} / \mathrm{kg}$ ethanol: $p<0.0001 ; 3 \mathrm{~g} / \mathrm{kg}$ ethanol: $p<0.0001)$ genotypes (Figures 1D and 2). In the LC, a significant impact of genotype $(\mathrm{F}(2,57)=9.648, p=0.0002)$, dose $(F(2,57)=6.596, p=0.0027)$, and an interaction of genotype $\times$ dose $(F(4,57)=3.137, p=0.0212)$ were detected. Post hoc analysis found a significant increase in c-Fos expression relative to saline injection in the HS ( $2 \mathrm{~g} / \mathrm{kg}$ ethanol: $p=0.4805 ; 3 \mathrm{~g} / \mathrm{kg}$ ethanol: $p=0.0257)$ and iHDID2 $(2 \mathrm{~g} / \mathrm{kg}$ ethanol: $p=0.0076 ; 3 \mathrm{~g} / \mathrm{kg}$ ethanol: $p=0.0013)$, but not in the iHDID1 $(2 \mathrm{~g} / \mathrm{kg}$ ethanol: $p=0.9044 ; 3 \mathrm{~g} / \mathrm{kg}$ ethanol: $p=0.8550)$ genotypes (Figures $1 \mathrm{E}$ and 3$)$. In the PBN, a significant impact of dose $(\mathrm{F}(2,62)=22.12$ 
$p<0.0001)$, but not genotype $(\mathrm{F}(2,62)=0.1425, p=0.8675)$ nor an interaction $(\mathrm{F}(4,62)=0.08052$, $p=0.9880)$ was detected. Post hoc analysis found a significant increase in c-Fos expression relative to saline injection in the HS ( $2 \mathrm{~g} / \mathrm{kg}$ ethanol: $p=0.0030 ; 3 \mathrm{~g} / \mathrm{kg}$ ethanol: $p=0.0159), \mathrm{iHDID} 1$ (2 g/kg ethanol: $p=0.0135 ; 3 \mathrm{~g} / \mathrm{kg}$ ethanol: $p=0.0022)$, and iHDID2 ( $\mathrm{g} / \mathrm{kg}$ ethanol: $p=0.0015 ; 3 \mathrm{~g} / \mathrm{kg}$ ethanol: $p=0.0010$ ) genotypes (Figures $1 \mathrm{~F}$ and 4 ).

A
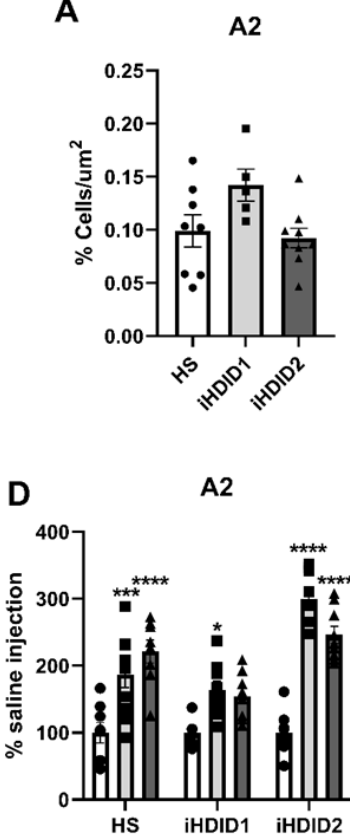

B

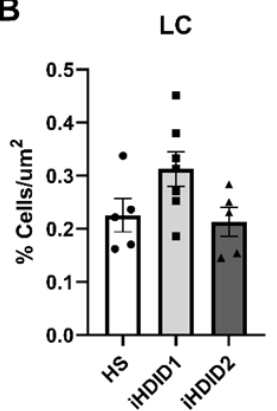

LC

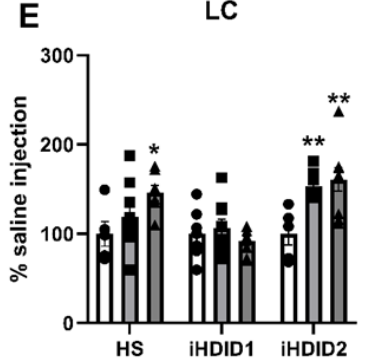

C PBN
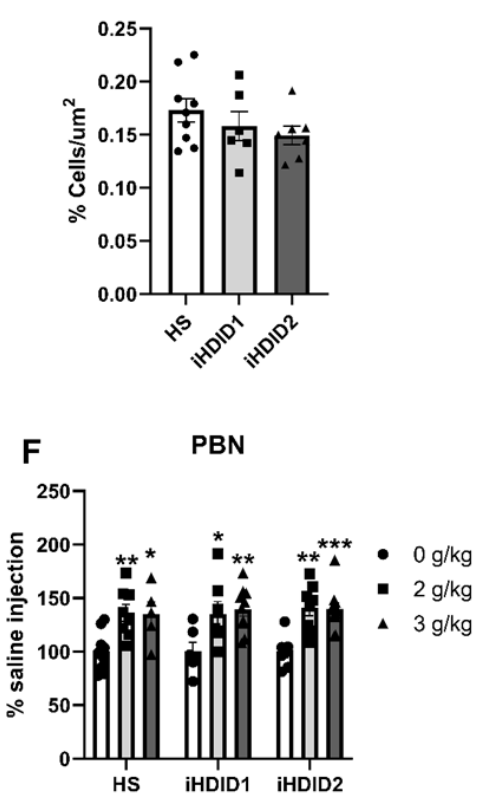

Figure 1. C-Fos Levels in the Hindbrain. (A-C) No difference in saline-induced c-Fos activation was detected between genotypes in any area. (D) Ethanol induced increased c-Fos levels in the A2 at all doses in the HS and iHDID2, but not at the $3 \mathrm{~g} / \mathrm{kg}$ dose in the iHDID1 line. (E) Ethanol induced changes in c-Fos levels relative to saline in the HS and iHDID2, but not iHDID1 in the LC. (F) Ethanol induced increased c-Fos levels in the PBN in all genotypes. ${ }^{*} p<0.05,{ }^{* *} p<0.01,{ }^{* * *} p<0.001,{ }^{* * * *} p<0.0001$ from two-way ANOVA Tukey's multiple comparisons test. 


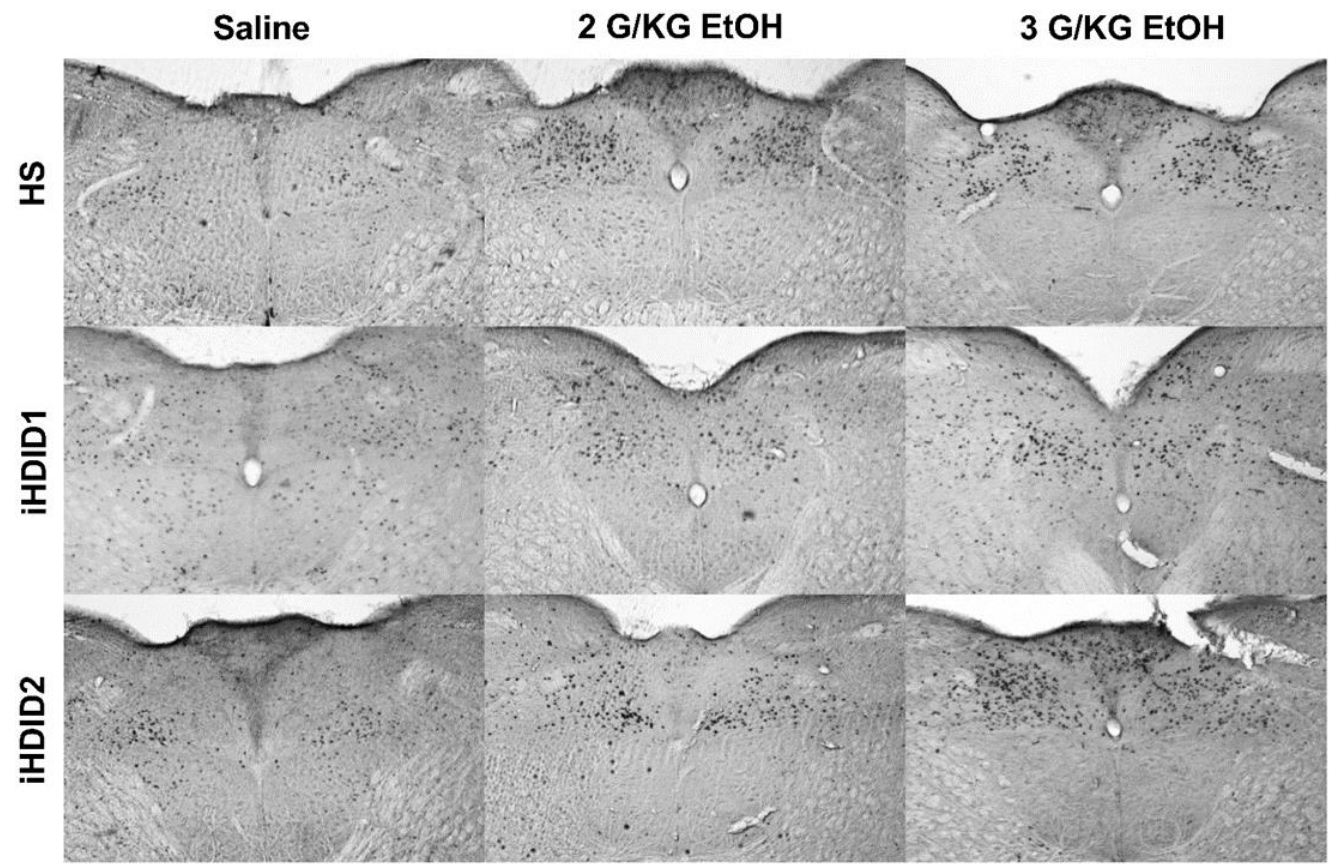

Figure 2. A2 Region of the Solitary Tract Nucleus. Representative images of c-Fos expression in the A2 across genotype and treatment types.

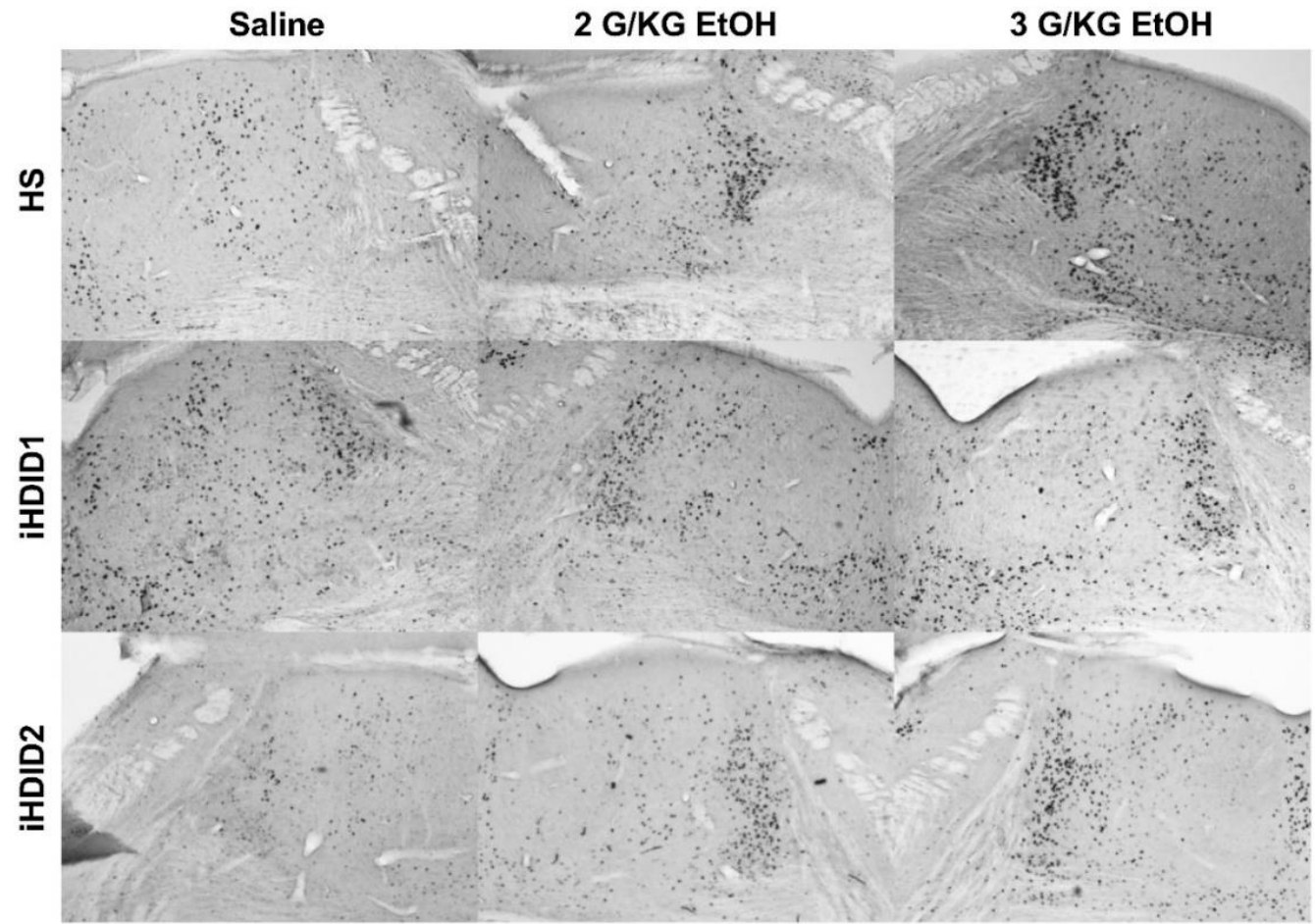

Figure 3. Locus Coeruleus. Representative images of c-Fos expression in the LC across genotype and treatment types. 


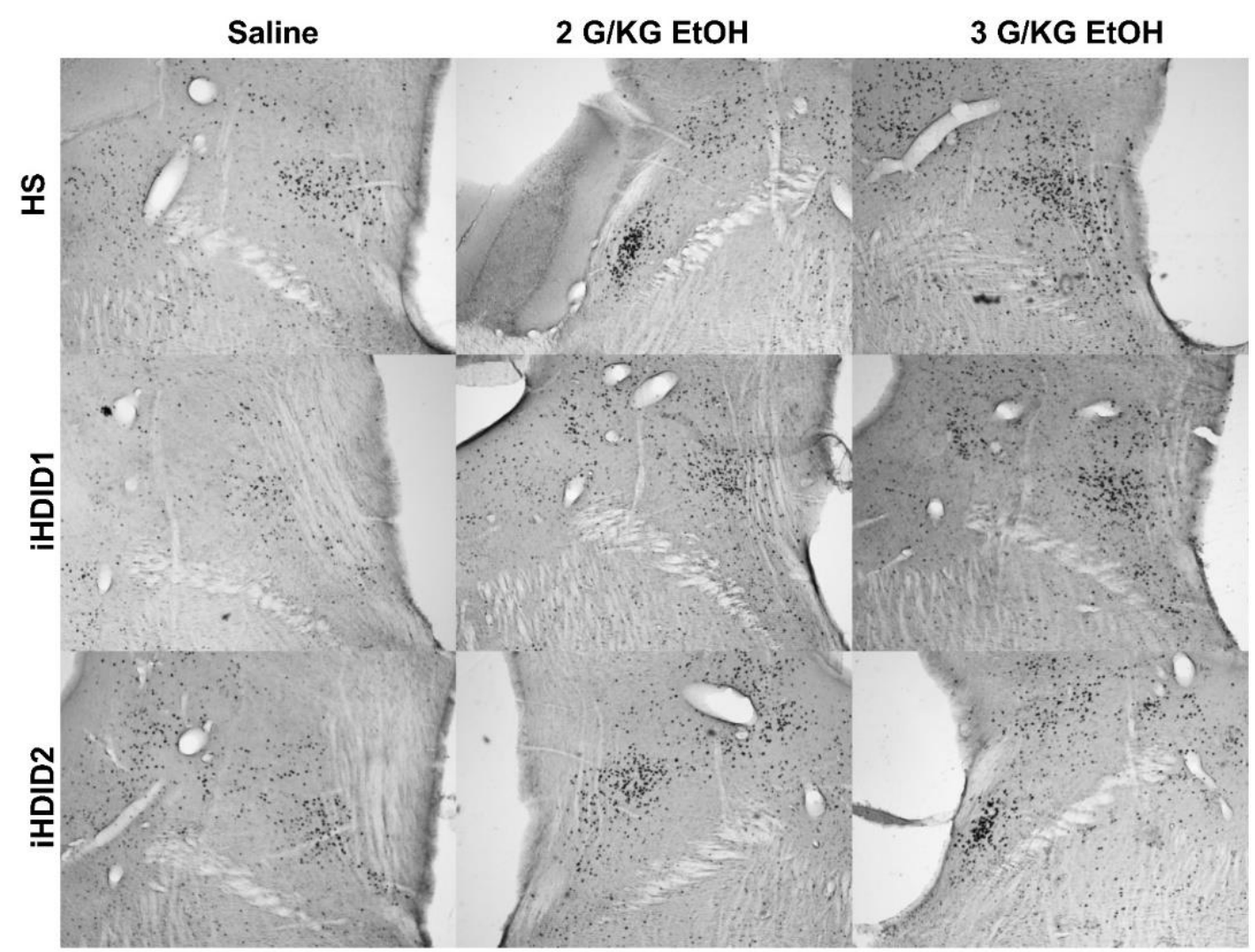

Figure 4. Parabrachial Nucleus. Representative images of c-Fos expression in the PBN across genotype and treatment types.

\subsection{C-Fos Response in the Extended Amygdala}

Central amygdala (CeA) and basolateral amygdala (BLA) numbers: HS saline, $n=6(\mathrm{~F}=3, \mathrm{M}=3)$, $2 \mathrm{~g} / \mathrm{kg}, n=7(\mathrm{~F}=2, \mathrm{M}=5), 3 \mathrm{~g} / \mathrm{kg}, n=9(\mathrm{~F}=5, \mathrm{M}=4) ; \mathrm{iHDID} 1$ saline, $n=7(\mathrm{~F}=3, \mathrm{M}=4), 2 \mathrm{~g} / \mathrm{kg}$, $n=10(\mathrm{~F}=5, \mathrm{M}=5), 3 \mathrm{~g} / \mathrm{kg}, n=8(\mathrm{~F}=4, \mathrm{M}=4) ; \mathrm{iHDID} 2$ saline, $n=8(\mathrm{~F}=5, \mathrm{M}=3), 2 \mathrm{~g} / \mathrm{kg}, n=6(\mathrm{~F}=3$, $\mathrm{M}=3), 3 \mathrm{~g} / \mathrm{kg}, n=8(\mathrm{~F}=4, \mathrm{M}=4)$. bed nucleus of the stria terminalis (BNST) numbers: HS saline, $n=5(\mathrm{~F}=2, \mathrm{M}=3), 2 \mathrm{~g} / \mathrm{kg}, n=6(\mathrm{~F}=3, \mathrm{M}=3), 3 \mathrm{~g} / \mathrm{kg}, n=7(\mathrm{~F}=5, \mathrm{M}=2) ;$ iHDID1 saline, $n=5(\mathrm{~F}=3$, $\mathrm{M}=2), 2 \mathrm{~g} / \mathrm{kg}, n=7(\mathrm{~F}=3, \mathrm{M}=4), 3 \mathrm{~g} / \mathrm{kg}, n=5(\mathrm{~F}=2, \mathrm{M}=3)$; iHDID2 saline, $n=7(\mathrm{~F}=4, \mathrm{M}=3)$, $2 \mathrm{~g} / \mathrm{kg}, n=7(\mathrm{~F}=3, \mathrm{M}=4), 3 \mathrm{~g} / \mathrm{kg}, n=6(\mathrm{~F}=4, \mathrm{M}=2)$.

C-Fos immunoreactivity following injection of 0 (saline) was assessed by one-way ANOVA and Dunnett's post hoc test in male and female HS, iHDID1, and iHDID2 mice to assess potential baseline differences in regional activity in response to injection. One-way ANOVA detected no significant differences between genotypes in the BLA $(F(2,18)=2.324, p=0.1265)$, CeA $(F(2,18)=1.146$, $p=0.3399)$, or BNST $(\mathrm{F}(2,14)=1.614, p=0.2341)$ (Figure $5 \mathrm{~A}-\mathrm{C})$. 
A

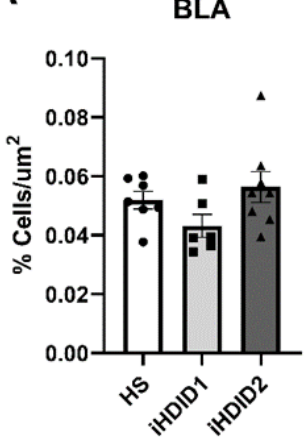

D

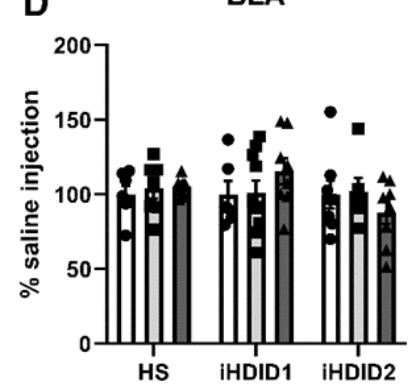

B
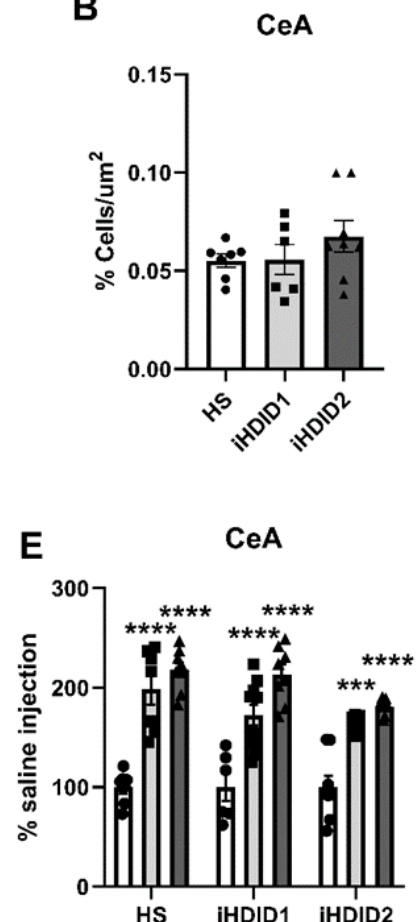

C BNST

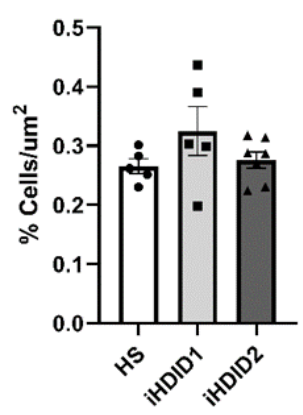

$\mathbf{F}$

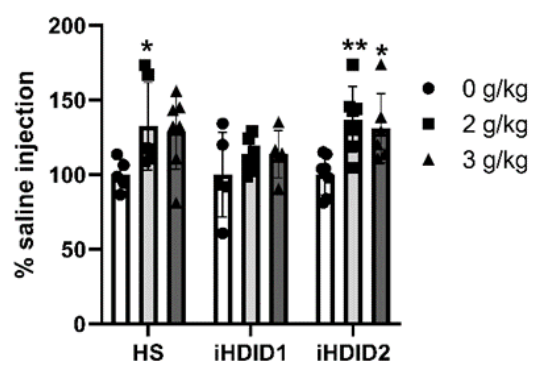

Figure 5. C-Fos Levels in the Extended Amygdala. (A-C) No difference in saline-induced c-Fos activation was detected between genotypes in any area. (D) Ethanol induced no change in c-Fos levels relative to saline in any genotype in the BLA. (E) Ethanol induced robust changes in c-Fos levels relative to saline in any genotype in the CeA. (F) Ethanol induced increased c-Fos levels in the BNST in the HS and iHDID2, but not iHDID1 line. ${ }^{*} p<0.05,{ }^{* *} p<0.01,{ }^{* * *} p<0.001$, and ${ }^{* * * *} p<0.0001$ from two-way ANOVA Tukey's multiple comparisons test.

The change in percent c-Fos expression relative to the saline injection was then assessed following a 2 or $3 \mathrm{~g} / \mathrm{kg}$ ethanol injection with two-way ANOVA (genotype $\times$ dose) and Tukey's multiple comparisons test. In the BLA, no significant impact of genotype $(\mathrm{F}(2,59)=1.011, p=0.3700)$, dose $(\mathrm{F}(2,59)=0.1134$, $p=0.8930)$, or interaction $(\mathrm{F}(4,59)=1.128, p=0.3521)$ was detected (Figures 5D and 6). In the CeA, a significant impact of genotype $(\mathrm{F}(2,59)=3.855, p=0.0267)$ and dose $(\mathrm{F}(2,59)=84.06, p<0.0001)$, but not an interaction $(\mathrm{F}(4,59)=1.487, p=0.2176)$, were detected. Post hoc analysis found a significant increase in c-Fos expression relative to saline injection in the HS $(2 \mathrm{~g} / \mathrm{kg}$ ethanol: $p<0.0001 ; 3 \mathrm{~g} / \mathrm{kg}$ ethanol: $p<0.0001)$, iHDID1 ( $2 \mathrm{~g} / \mathrm{kg}$ ethanol: $p<0.0001 ; 3 \mathrm{~g} / \mathrm{kg}$ ethanol: $p<0.0001)$, and iHDID2 ( $2 \mathrm{~g} / \mathrm{kg}$ ethanol: $p=0.0001 ; 3 \mathrm{~g} / \mathrm{kg}$ ethanol: $p<0.0001$ ) genotypes (Figures $5 \mathrm{E}$ and 6$)$. In the BNST, a significant impact of dose $(\mathrm{F}(2,46)=8.907, p=0.0005)$, but not genotype $(\mathrm{F}(2,46)=2.233, p=0.1187)$ nor an interaction $(\mathrm{F}(4,46)=0.6160, p=0.6533)$ was detected. Post hoc analysis found a significant increase in c-Fos expression relative to saline injection in the HS (2 g/ $\mathrm{kg}$ ethanol: $p=0.0368 ; 3 \mathrm{~g} / \mathrm{kg}$ ethanol: $p=0.0597)$ and iHDID2 $(2 \mathrm{~g} / \mathrm{kg}$ ethanol: $p=0.0052 ; 3 \mathrm{~g} / \mathrm{kg}$ ethanol: $p=0.0282)$, but not in iHDID1 ( $2 \mathrm{~g} / \mathrm{kg}$ ethanol: $p=0.5772 ; 3 \mathrm{~g} / \mathrm{kg}$ ethanol: $p=0.5560)$ genotypes (Figures 5F and 7). 


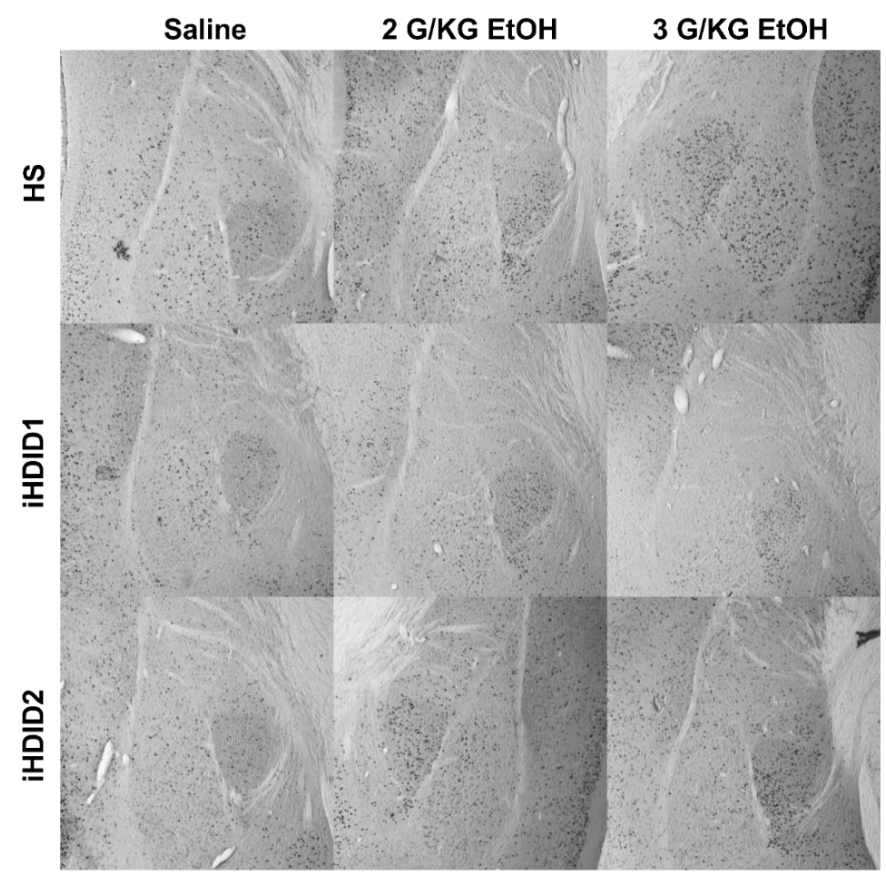

Figure 6. Basolateral and Central Amygdala. Representative images of c-Fos expression in the BLA and $\mathrm{CeA}$ across genotype and treatment types.

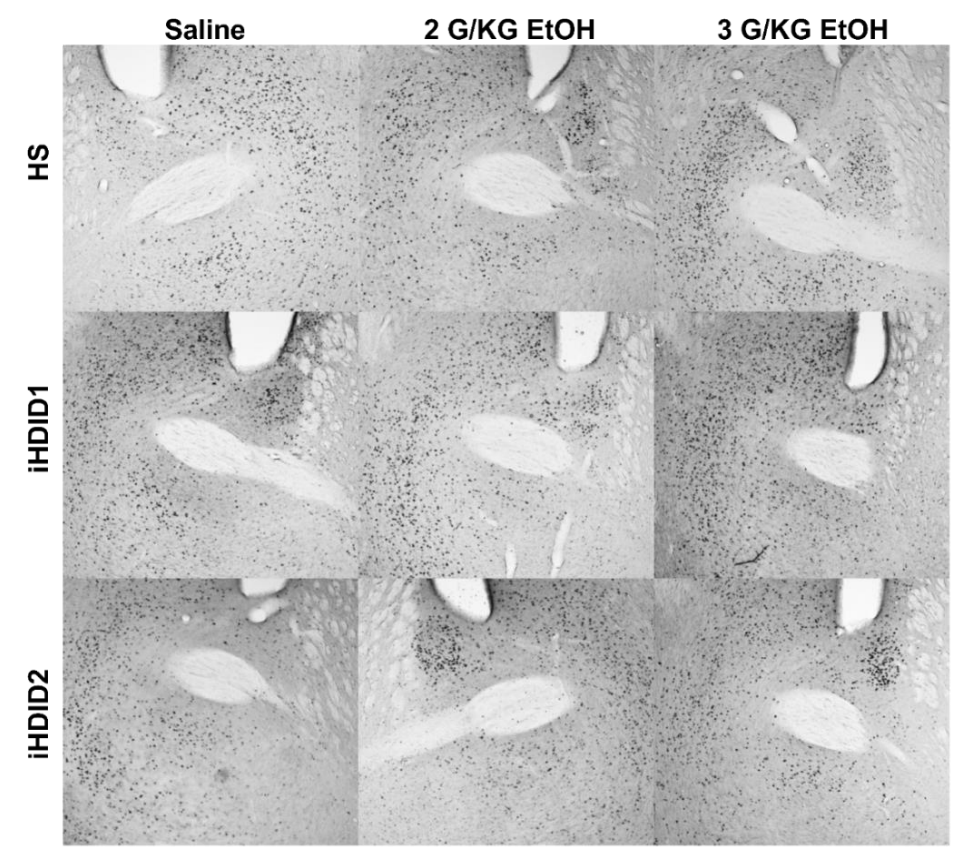

Figure 7. Bed Nucleus of the Stria Terminalis. Representative images of c-Fos expression in the BNST across genotype and treatment types.

\subsection{C-Fos Response in the Thalamic Area}

Lateral and medial habenula ( $\mathrm{LHb}$ and $\mathrm{MHb})$ numbers: HS saline, $n=8(\mathrm{~F}=5, \mathrm{M}=3), 2 \mathrm{~g} / \mathrm{kg}$, $n=6(\mathrm{~F}=3, \mathrm{M}=3), 3 \mathrm{~g} / \mathrm{kg}, n=7(\mathrm{~F}=4, \mathrm{M}=3) ;$ iHDID1 saline, $n=4(\mathrm{~F}=2, \mathrm{M}=2), 2 \mathrm{~g} / \mathrm{kg}, n=7(\mathrm{~F}=4$, $\mathrm{M}=3), 3 \mathrm{~g} / \mathrm{kg}, n=6(\mathrm{~F}=2, \mathrm{M}=4) ;$ iHDID2 saline, $n=6(\mathrm{~F}=3, \mathrm{M}=3), 2 \mathrm{~g} / \mathrm{kg}, n=3(\mathrm{~F}=2, \mathrm{M}=1)$, $3 \mathrm{~g} / \mathrm{kg}, n=7(\mathrm{~F}=4, \mathrm{M}=3)$. Paraventricular nucleus of the thalamus (PVT) numbers: HS saline, $n=7$ $(\mathrm{F}=4, \mathrm{M}=3), 2 \mathrm{~g} / \mathrm{kg}, n=6(\mathrm{~F}=3, \mathrm{M}=3), 3 \mathrm{~g} / \mathrm{kg}, n=7(\mathrm{~F}=5, \mathrm{M}=2) ;$ iHDID1 saline, $n=7(\mathrm{~F}=3$, $\mathrm{M}=4), 2 \mathrm{~g} / \mathrm{kg}, n=9(\mathrm{~F}=4, \mathrm{M}=5), 3 \mathrm{~g} / \mathrm{kg}, n=6(\mathrm{~F}=2, \mathrm{M}=4)$; iHDID2 saline, $n=7(\mathrm{~F}=3, \mathrm{M}=4)$, $2 \mathrm{~g} / \mathrm{kg}, n=4(\mathrm{~F}=2, \mathrm{M}=2), 3 \mathrm{~g} / \mathrm{kg}, n=8(\mathrm{~F}=4, \mathrm{M}=4)$. 
C-Fos immunoreactivity following injection of 0 (saline) was assessed by one-way ANOVA and Dunnett's post hoc test in male and female HS, iHDID1, and iHDID2 mice to assess potential baseline differences in regional activity in response to injection. One-way ANOVA detected no significant differences between genotypes in the $\mathrm{LHb}(\mathrm{F}(2,15)=1.374, p=0.2831)$. One-way ANOVA detected a significant difference between genotypes in the $\mathrm{MHb}(\mathrm{F}(2,15)=9.650, p=0.0020)$ in which post hoc revealed a significant difference between HS and iHDID2 $(p=0.0014)$, but not iHDID1 $(p=0.8408)$. One-way ANOVA detected a significant difference between genotypes in the PVT $(F(2,18)=5.832$, $p=0.0112)$ in which post hoc revealed a significant difference between HS and iHDID2 $(p=0.0220)$, but not iHDID1 $(p=0.9389)$ (Figure 8A-C).

A
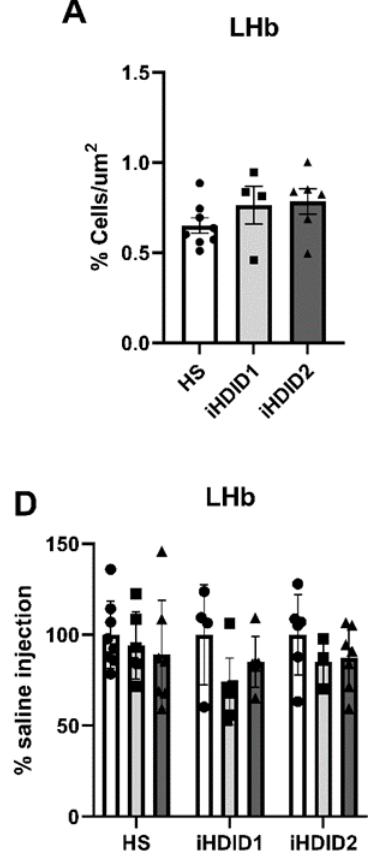

B
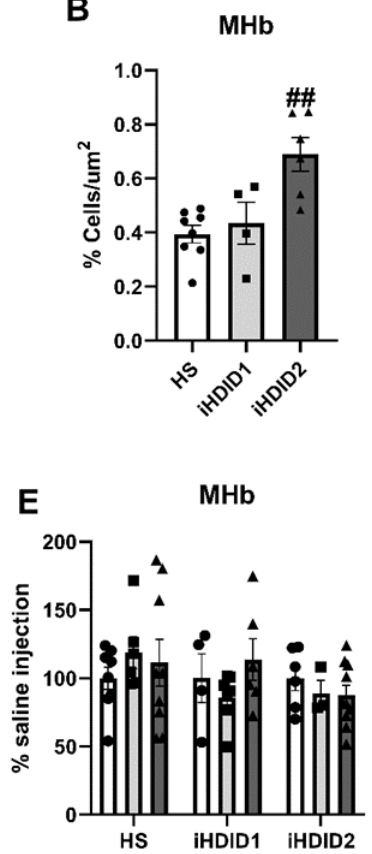
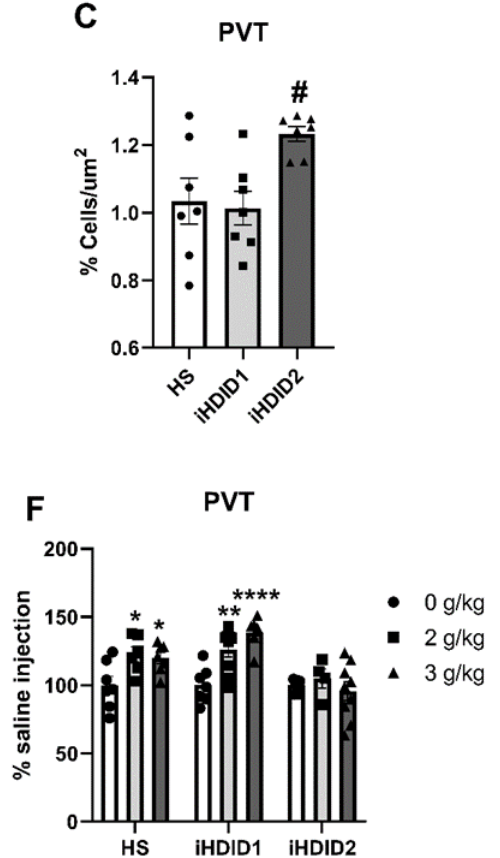

Figure 8. C-Fos Levels in the Midbrain. (A) No difference in saline-induced c-Fos activation was detected between genotypes in the $\mathrm{LHb}$. (B,C) A significant increase in saline-induced c-Fos levels were detected in the iHDID2, but not iHDID1, line in the MHb and PVT relative to the HS genotype. $(\mathrm{D}, \mathrm{E})$ Ethanol induced no change in c-Fos levels relative to saline in any genotype in the $\mathrm{LHb}$ or $\mathrm{MHb}$. (F) Ethanol induced increased c-Fos levels in the PVT in the HS and iHDID1, but not iHDID2 line. $\# p<0.05$, \#\# $p<0.01$ from one-way ANOVA Dunnett's multiple comparisons test; ${ }^{*} p<0.05$, ${ }^{* *} p<0.01$, and ${ }^{* * * *} p<0.0001$ from two-way ANOVA Tukey's multiple comparisons test.

The change in percent c-Fos expression relative to the saline injection was then assessed following a 2 or $3 \mathrm{~g} / \mathrm{kg}$ ethanol injection with two-way ANOVA (genotype $\times$ dose) and Tukey's multiple comparisons test. In the $\mathrm{LHb}$, no significant impact of genotype $(\mathrm{F}(2,45)=1.002, p=0.3754)$, dose $(\mathrm{F}(2,45)=2.975$, $p=0.0612)$, nor an interaction $(\mathrm{F}(4,45)=0.6694, p=0.6166)$ was detected (Figures $8 \mathrm{D}$ and 9$)$. In the $\mathrm{MHb}$, no significant impact of genotype $(\mathrm{F}(2,50)=1.496, p=0.2338)$, dose $(\mathrm{F}(2,50)=0.1997, p=0.8196)$, nor an interaction $(\mathrm{F}(4,50)=1.015, p=0.4089)$ was detected (Figures 8E and 9$)$. In the PVT, a significant effect of genotype $(F(2,53)=10.67, p=0.0001)$, dose $(F(2,53)=10.41, p=0.0002)$ and an interaction of genotype $\times$ se $(F(4,53)=4.038, p=0.0062)$ were detected. Post hoc analysis found a significant increase in c-Fos expression relative to saline injection in the HS (2 g/ $/ \mathrm{kg}$ ethanol: $p=0.0193 ; 3 \mathrm{~g} / \mathrm{kg}$ ethanol: $p=0.0358)$, iHDID1 ( $2 \mathrm{~g} / \mathrm{kg}$ ethanol: $p=0.0024 ; 3 \mathrm{~g} / \mathrm{kg}$ ethanol: $p<0.0001)$, but not iHDID2 ( $2 \mathrm{~g} / \mathrm{kg}$ ethanol: $p=0.8514 ; 3 \mathrm{~g} / \mathrm{kg}$ ethanol: $p=0.8304$ ) genotypes (Figures $8 \mathrm{~F}$ and 9 ). 


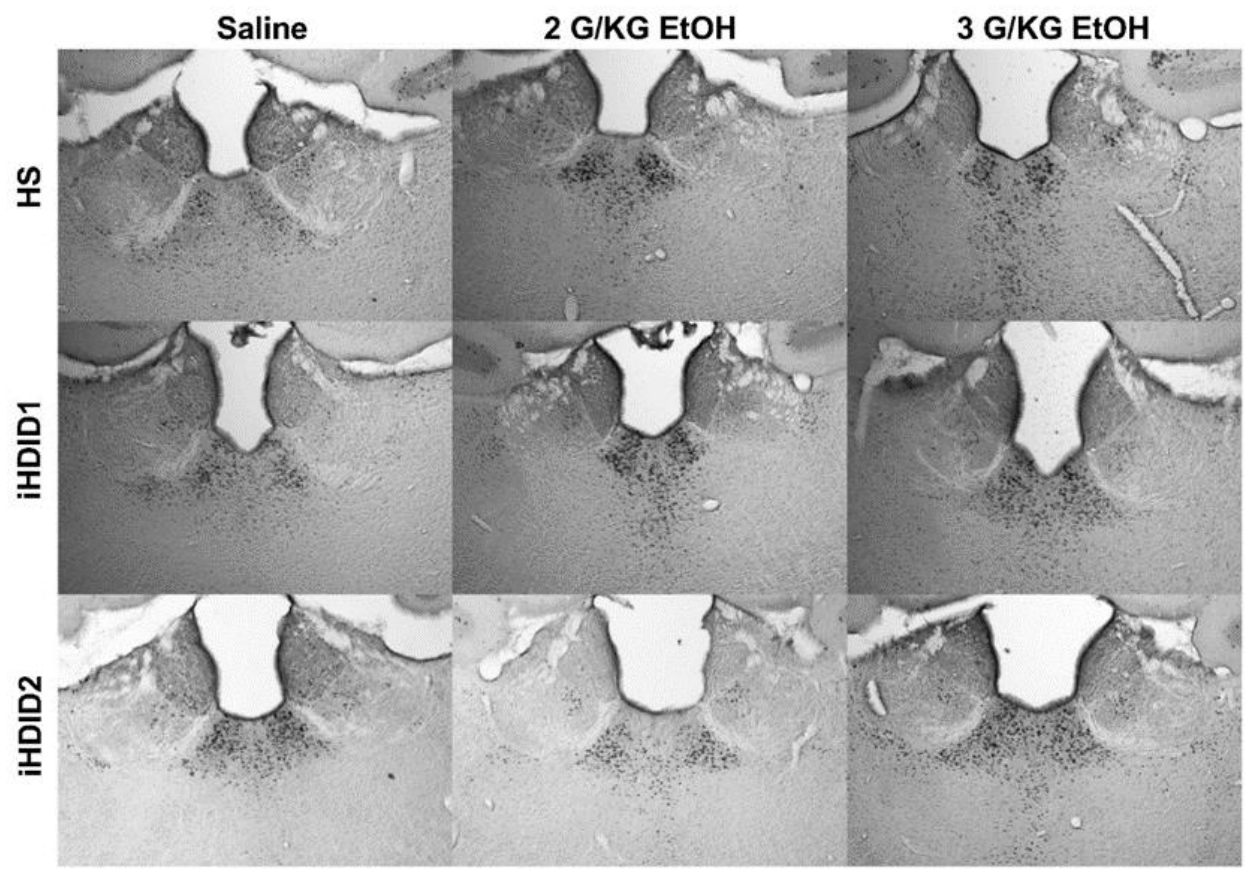

Figure 9. Lateral and Medial Habenula and Paraventricular Nucleus of the Thalamus. Representative images of c-Fos expression in the $\mathrm{LHb}, \mathrm{MHb}$, and PVT across genotype and treatment types.

\subsection{C-Fos Response in Other Regions Examined}

Periaqueductal gray (PAG): HS saline, $n=7(\mathrm{~F}=4, \mathrm{M}=3), 2 \mathrm{~g} / \mathrm{kg}, n=5(\mathrm{~F}=3, \mathrm{M}=2), 3 \mathrm{~g} / \mathrm{kg}$, $n=8(\mathrm{~F}=4, \mathrm{M}=4) ; \mathrm{iHDID} 1$ saline, $n=5(\mathrm{~F}=2, \mathrm{M}=3), 2 \mathrm{~g} / \mathrm{kg}, n=8(\mathrm{~F}=3, \mathrm{M}=5), 3 \mathrm{~g} / \mathrm{kg}, n=6(\mathrm{~F}=3$, $\mathrm{M}=3)$; iHDID2 saline, $n=7(\mathrm{~F}=3, \mathrm{M}=4), 2 \mathrm{~g} / \mathrm{kg}, n=6(\mathrm{~F}=2, \mathrm{M}=4), 3 \mathrm{~g} / \mathrm{kg}, n=10(\mathrm{~F}=5, \mathrm{M}=5)$. Edinger-Westphal nuclei (EW) numbers: HS saline, $n=6(\mathrm{~F}=4, \mathrm{M}=2), 2 \mathrm{~g} / \mathrm{kg}, n=4(\mathrm{~F}=3, \mathrm{M}=2)$, $3 \mathrm{~g} / \mathrm{kg}, n=9(\mathrm{~F}=5, \mathrm{M}=4)$; iHDID1 saline, $n=5(\mathrm{~F}=3, \mathrm{M}=2), 2 \mathrm{~g} / \mathrm{kg}, n=9(\mathrm{~F}=7, \mathrm{M}=2), 3 \mathrm{~g} / \mathrm{kg}, n=6$ $(\mathrm{F}=3, \mathrm{M}=3)$; iHDID2 saline, $n=6(\mathrm{~F}=2, \mathrm{M}=4), 2 \mathrm{~g} / \mathrm{kg}, n=6(\mathrm{~F}=3, \mathrm{M}=3), 3 \mathrm{~g} / \mathrm{kg}, n=10(\mathrm{~F}=5$, $\mathrm{M}=5)$. Rostromedial tegmental nucleus (RMTg) numbers: HS saline, $n=9(\mathrm{~F}=5, \mathrm{M}=4), 2 \mathrm{~g} / \mathrm{kg}, n=8$ $(\mathrm{F}=4, \mathrm{M}=4), 3 \mathrm{~g} / \mathrm{kg}, n=8(\mathrm{~F}=5, \mathrm{M}=3) ;$ iHDID1 saline, $n=7(\mathrm{~F}=3, \mathrm{M}=4), 2 \mathrm{~g} / \mathrm{kg}, n=10(\mathrm{~F}=5$, $\mathrm{M}=5), 3 \mathrm{~g} / \mathrm{kg}, n=6(\mathrm{~F}=2, \mathrm{M}=4) ;$ iHDID2 saline, $n=7(\mathrm{~F}=4, \mathrm{M}=3), 2 \mathrm{~g} / \mathrm{kg}, n=6(\mathrm{~F}=3, \mathrm{M}=3)$, $3 \mathrm{~g} / \mathrm{kg}, n=10(\mathrm{~F}=5, \mathrm{M}=5)$.

C-Fos immunoreactivity following injection of 0 (saline) was assessed by one-way ANOVA and Dunnett's post hoc test in male and female HS, iHDID1, and iHDID2 mice to assess potential baseline differences in regional activity in response to injection. One-way ANOVA detected no significant differences between genotypes in the PAG $(F(2,16)=0.2242, p=0.8016)$. One-way ANOVA detected a significant difference between genotypes in the $\operatorname{EW~}(\mathrm{F}(2,16)=12.37, p=0.0006)$ in which post hoc revealed a significant difference between HS and iHDID1 $(p=0.0011)$, but not iHDID2 $(p=0.9611)$. One-way ANOVA detected a significant difference between genotypes in the $\operatorname{RMTg}(\mathrm{F}(2,20)=4.018$, $p=0.0341)$ in which post hoc revealed a significant difference between HS and iHDID1 $(p=0.0296)$, but not iHDID2 ( $p=0.0906)$ (Figure 10A-C). 

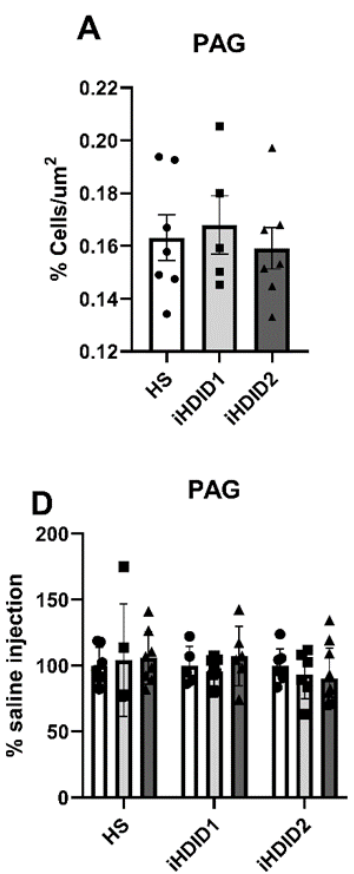
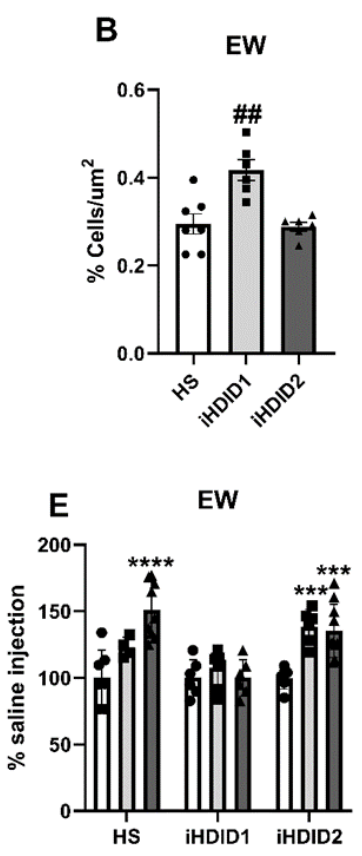
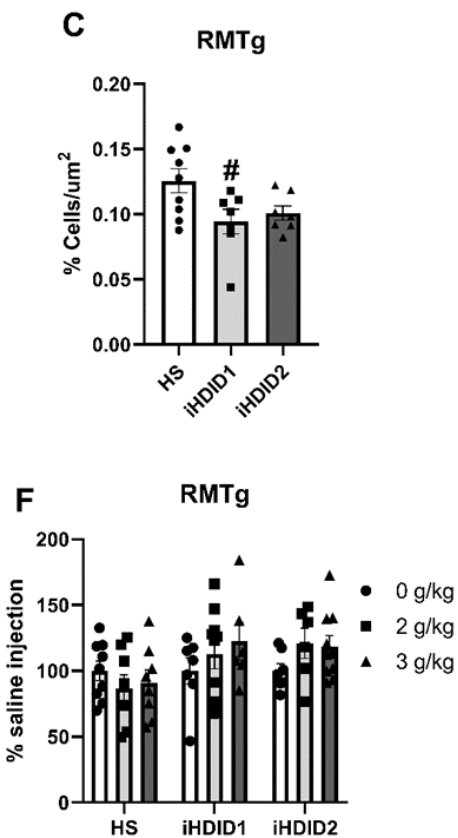

Figure 10. C-Fos Levels in Other Regions Examined. (A) No difference in saline-induced c-Fos activation was detected between genotypes in the PAG. (B,C) A significant difference in saline-induced c-Fos levels were detected in the iHDID1, but not iHDID2, line in the EW and RMTg relative to the HS genotype. (D) Ethanol induced no change in c-Fos levels relative to saline in any genotype in the PAG. (E) Ethanol induced changes in c-Fos levels relative to saline in the HS and HDID2, but not iHDID1 in the EW. (F) Ethanol induced no change in c-Fos levels relative to saline in any genotype in the RMTg. \# $p<0.05, \# \# p<0.01$ from one-way ANOVA Dunnett's multiple comparisons test; ${ }^{* * *} p<0.001$, and ${ }^{* * * *} p<0.0001$ from two-way ANOVA Tukey's multiple comparisons test.

The change in percent c-Fos expression relative to the saline injection was then assessed following a 2 or $3 \mathrm{~g} / \mathrm{kg}$ ethanol injection with two-way ANOVA (genotype $\times$ dose) and Tukey's multiple comparisons test. In the PAG, no significant impact of genotype $(\mathrm{F}(2,53)=0.9948, p=0.3766)$, dose $(\mathrm{F}(2,53)=0.1637$, $p=0.8494)$, nor an interaction $(\mathrm{F}(4,53)=0.5041, p=0.7329)$ was detected (Figures 10D and 11). In the $\mathrm{EW}$, a significant effect of genotype $(\mathrm{F}(2,53)=12.00, p<0.0001)$, dose $(\mathrm{F}(2,53)=17.41, p<0.0001)$, and an interaction of genotype $\times$ dose $(F(4,53)=5.277, p=0.0012)$ was detected. Post hoc analysis found a significant increase in c-Fos expression relative to saline injection in the HS $(2 \mathrm{~g} / \mathrm{kg}$ ethanol: $p=0.0740$; $3 \mathrm{~g} / \mathrm{kg}$ ethanol: $p<0.0001)$ and iHDID2 $(2 \mathrm{~g} / \mathrm{kg}$ ethanol: $p=0.0004 ; 3 \mathrm{~g} / \mathrm{kg}$ ethanol: $p=0.0003)$, but not iHDID1 ( $2 \mathrm{~g} / \mathrm{kg}$ ethanol: $p=0.6829 ; 3 \mathrm{~g} / \mathrm{kg}$ ethanol: $p>0.9999)$ genotypes (Figures 10E and 12). In the RMTg, a significant impact of genotype $(\mathrm{F}(2,62)=4.122, p=0.0208)$ but not dose $(\mathrm{F}(2,62)=0.8829$, $p=0.4187)$ nor an interaction $(\mathrm{F}(4,62)=1.095, p=0.3668)$ was detected. Tukey's multiple comparisons test detected no significant differences in any genotype $(p>0.05)$ (Figures $10 \mathrm{~F}$ and 13$)$. 


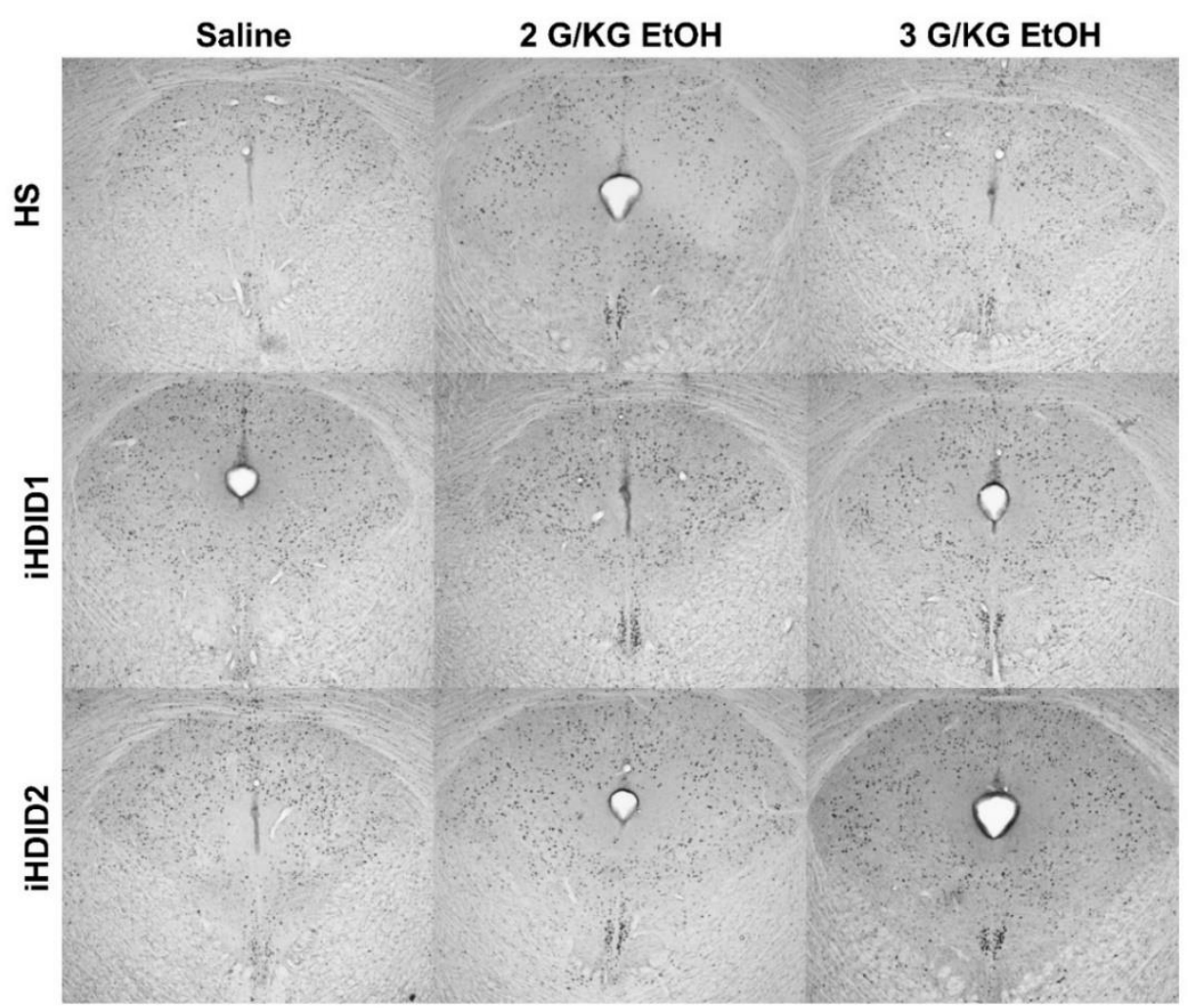

Figure 11. Periaqueductal Gray. Representative images of c-Fos expression in the PAG across genotype and treatment types.

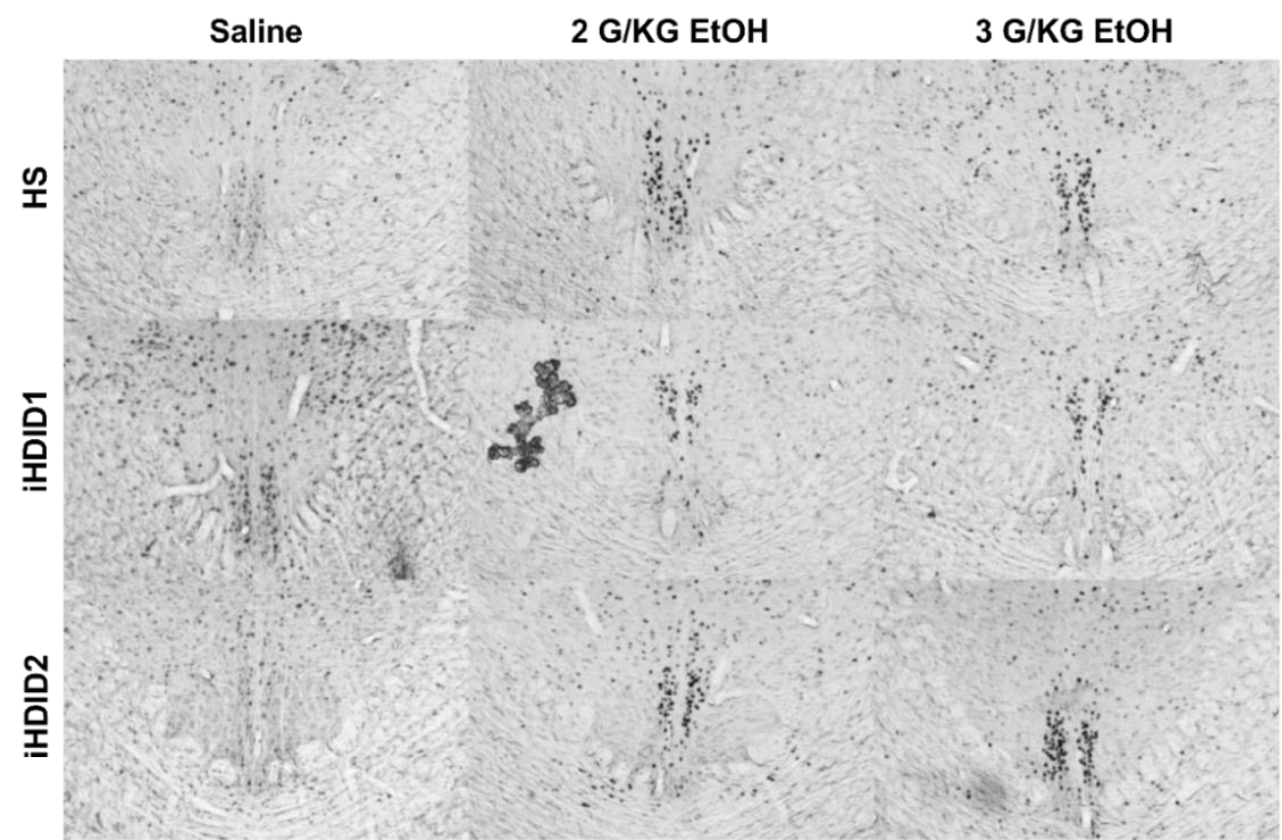

Figure 12. Edinger Westphal Nucleus. Representative images of c-Fos expression in the EW across genotype and treatment types. 


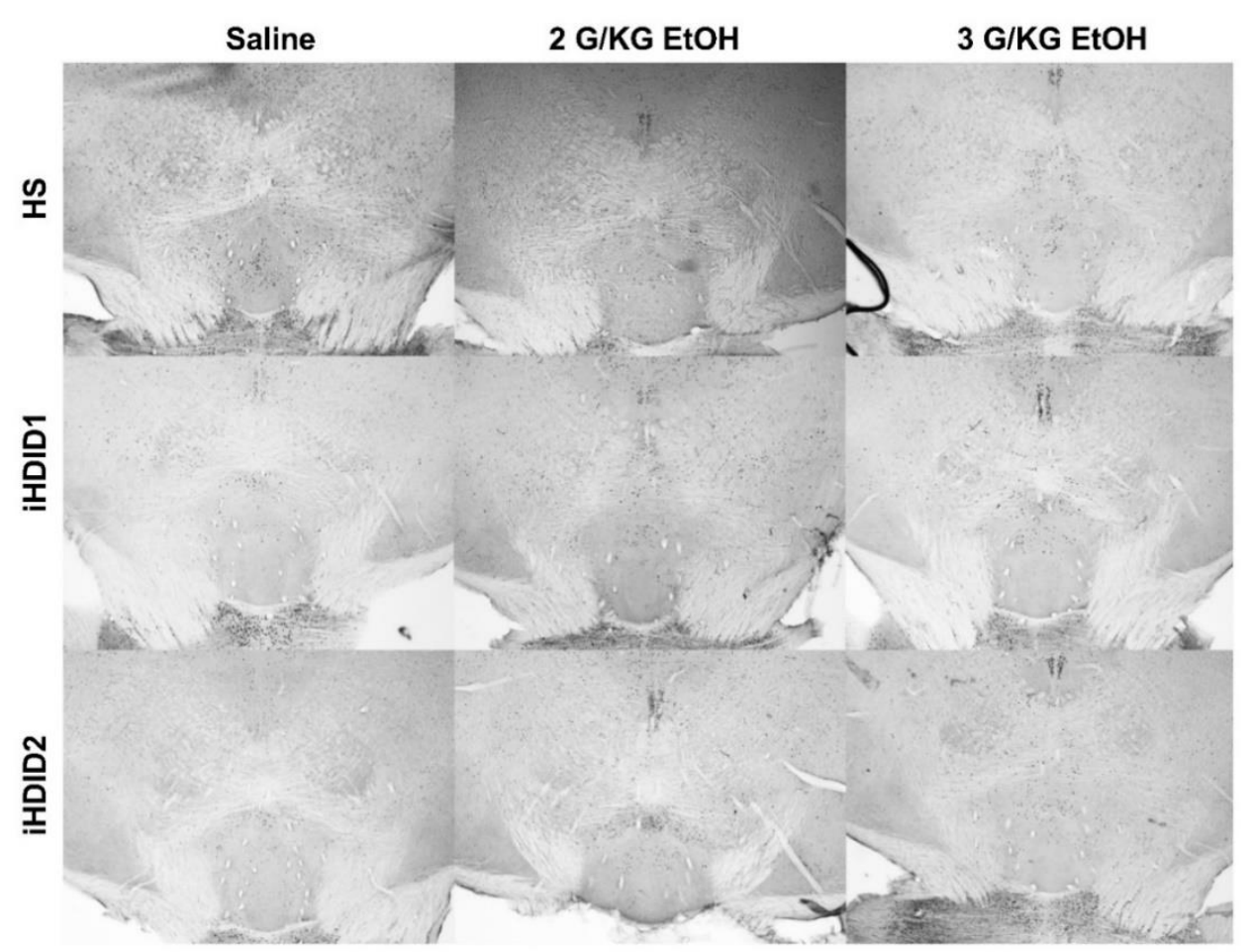

Figure 13. Rostromedial Tegmental Nucleus. Representative images of c-Fos expression in the RMTg across genotype and treatment types.

\section{Discussion}

In this work, we have demonstrated both overlapping and significantly divergent patterns of ethanol-induced c-Fos activation in the iHDID1 and iHDID2 lines of mice, relative to their HS parent strain (see Table 1 for summary of results). These findings complement previous work demonstrating an extensive difference in patterns of c-Fos activation in genetic lines bred for a high ethanol preference relative to low-preference lines $[10,11,15]$. Notably, c-Fos activation in select regions differed between the iHDID1 and iHDID2 lines, lending strength to the hypothesis that polygenetic factors underlie the same behavioral end point in these two replicate inbred lines, as previously observed in assessment of brain gene coexpression networks of the founding HDID1 and HDID2 strains [6]. Specifically, c-Fos activation in the iHDID1 line was found to differ from the HS line either at baseline or following ethanol exposure in the BNST, A2, LC, EW, and to a lesser extent the RMTg. In contrast, the HDID2 line differed compared to the HS line in the PVT and MHb. It is notable that while the iHDID1 and iHDID2 lines showed similar c-Fos activity as the HS line in multiple brain regions, in no region did the two both significantly differ from the HS line together. An important limitation to the present work is that there was not sufficient sample size to detect potential sex differences ethanol-induced effects on c-Fos expression, and it will be important to address this variable in future research. Below we provided additional consideration for differences in ethanol-induced c-Fos expression that we observed between these lines. It is of note the iHDID lines were bred specifically for binge-ethanol behavior, which cannot be generalized to other forms of intake. Indeed, the HDID1 line has been found to ingest lower amounts of high concentration ethanol relative to the HS founder line [16], highlighting that an increase in one form of ethanol intake in no way suggests an increase in all ethanol-directed behaviors. Given this caveat, we include literature related to genetic lines bred for overall higher ethanol intake/preference to offer additional context in discussion and to note brain regions involved in binge-ethanol intake may be critical across multiple stages/patterns of ethanol intoxication. 
Table 1. Summary of significant differences in vehicle- or ethanol-induced c-Fos expression between genotypes. Arrow indicates increase $(\uparrow)$ or decrease $(\downarrow)$ in c-Fos expression. A2: A2 region of the nucleus of tract solitary (NTS); LC: locus coeruleus; PBN: parabrachial nucleus; BLA: basolateral amygdala; CeA: central amygdala; BNST: bed nucleus of the stria terminals; LHb: lateral habenula; MHb: medial habenula; PVT: paraventricular nucleus of the thalamus; PAG: periaqueductal gray; EW: Edinger-Westphal nuclei; RMTg: rostromedial tegmental nucleus ${ }^{*} p<0.05,{ }^{* *} p<0.01,{ }^{* * *} p<0.001$, and ${ }^{* * * *} p<0.0001$ (see results for statistical analysis performed).

\begin{tabular}{|c|c|c|c|c|}
\hline Region & Genotype & $\begin{array}{c}\text { VEH c-Fos vs. HS } \\
\text { VEH }\end{array}$ & $\begin{array}{l}2 \mathrm{G} / \mathrm{KG} \text { EtOH } \\
\text { c-Fos vs. VEH }\end{array}$ & $\begin{array}{l}3 \mathrm{G} / \mathrm{KG} \text { EtOH } \\
\text { c-Fos vs. VEH }\end{array}$ \\
\hline \multirow[t]{3}{*}{ A2 } & HS & & $\uparrow^{* * *}$ & $\uparrow^{* * * *}$ \\
\hline & iHDID1 & - & $\uparrow^{*}$ & - \\
\hline & iHDID2 & - & $\uparrow^{* * * *}$ & $\uparrow^{* * * *}$ \\
\hline \multirow[t]{3}{*}{ LC } & HS & & - & $\uparrow^{*}$ \\
\hline & iHDID1 & - & - & - \\
\hline & iHDID2 & - & $\uparrow^{* *}$ & $\uparrow^{* *}$ \\
\hline \multirow[t]{3}{*}{ PBN } & HS & & $\uparrow^{* *}$ & $\uparrow^{*}$ \\
\hline & iHDID1 & - & $\uparrow^{*}$ & $\uparrow^{* *}$ \\
\hline & iHDID2 & - & $\uparrow^{* *}$ & $\uparrow^{* * *}$ \\
\hline \multirow[t]{3}{*}{ BLA } & HS & & - & - \\
\hline & iHDID1 & - & - & - \\
\hline & iHDID2 & - & - & - \\
\hline \multirow[t]{3}{*}{$\mathrm{CeA}$} & HS & & $\uparrow^{* * * *}$ & $\uparrow^{* * * *}$ \\
\hline & iHDID1 & - & $\uparrow^{* * * *}$ & $\uparrow^{* * * *}$ \\
\hline & iHDID2 & - & $\uparrow^{* * *}$ & $\uparrow^{* * * *}$ \\
\hline \multirow[t]{3}{*}{ BNST } & HS & & $\uparrow^{*}$ & - \\
\hline & iHDID1 & - & - & - \\
\hline & iHDID2 & - & $\uparrow^{* *}$ & $\uparrow^{*}$ \\
\hline \multirow[t]{3}{*}{ LHb } & HS & & - & - \\
\hline & iHDID1 & - & - & - \\
\hline & iHDID2 & - & - & - \\
\hline \multirow[t]{3}{*}{$\mathbf{M H b}$} & $\mathrm{HS}$ & & - & - \\
\hline & iHDID1 & - & - & - \\
\hline & iHDID2 & $\uparrow^{* *}$ & - & - \\
\hline \multirow[t]{3}{*}{ PVT } & HS & & $\uparrow^{*}$ & $\uparrow^{*}$ \\
\hline & iHDID1 & - & $\uparrow^{* *}$ & $\uparrow^{* * * *}$ \\
\hline & iHDID2 & $\uparrow^{*}$ & - & - \\
\hline \multirow[t]{3}{*}{ PAG } & $\mathrm{HS}$ & & - & - \\
\hline & iHDID1 & - & - & - \\
\hline & iHDID2 & - & - & - \\
\hline \multirow[t]{3}{*}{ EW } & HS & & - & $\uparrow^{* * * *}$ \\
\hline & iHDID1 & $\uparrow^{* *}$ & - & - \\
\hline & iHDID2 & - & $\uparrow^{* * *}$ & $\uparrow^{* * *}$ \\
\hline \multirow[t]{3}{*}{ RMTg } & HS & & - & - \\
\hline & iHDID1 & $\downarrow^{*}$ & - & - \\
\hline & iHDID2 & - & - & - \\
\hline
\end{tabular}

\subsection{Hindbrain Regions}

Previous work has demonstrated that iHDID lines exhibit decreased sensitivity to the aversive properties of ethanol when compared to the control lines, but do not show altered sensitivity to the rewarding properties of ethanol $[10,11,15]$. Based on these observations, the doses of ethanol used in the present work were specifically chosen to examine potential mechanisms underlying altered sensitivity to the aversive effects of ethanol. Specifically, we selected doses that have been shown 
to induced blunted ethanol-induced CTA in iHDID1 and iHDID2 relative to the parent HS line [4]. Hindbrain regions, included the nucleus of tract solitary (NTS) and LC, have been shown to be active in response to stimuli that can support CTA (for review see [17]). The involvement of the A2, a subregion of the NTS, in CTA is thought to be due to noradrenaline (NE) efferents to the PBN, a brain area that is strongly related to deleterious somatosensory and visceral insults [18] (see below). The A2 noradrenergic neurons further modulate seeking behaviors for drugs of abuse [19]. Previous work has found acute ethanol exposure induces A2 c-Fos expression in mice and rats [20-22]. Interestingly, while both ethanol doses induced increased c-Fos in the A2 in the HS and iHDID2 line, only the low dose of ethanol induced c-Fos activation in the iHDID1 line, suggesting that ethanol-induced activation of the A2 is blunted in iHDID1 mice. Given the role of the NTS in aversion learning, it is tempting to speculate that blunted ethanol-induced activation of the A2 in iHDID1 mice may contribute to their reduced sensitivity to the aversive effects of ethanol. However, since iHDID2 mice similarly show reduced sensitivity to the aversive effects of ethanol, a link between the A2 sensitivity to the aversive properties of ethanol is not straightforward. Regardless, blunted ethanol-induced c-Fos expression in the A2 of iHDID1 mice relative to the HS strain suggests a potential mechanism involved in high binge-like ethanol intake in the iHDID1 line.

The LC is a catecholamine nucleus and NE is its primary neurotransmitter [23]. With extensive projections throughout the central nervous system [24], this brainstem region has a major role in stress-related behaviors [25], and is involved in the shaping of alcohol-drinking behaviors [21,26]. It is known that aversive states induce LC activation, including ethanol in doses that support CTA [27-30]. Our findings show variations in the pattern of c-Fos expression on LC induced by acute ethanol, as the iHDID1 line mice displayed no LC activation, while iHDID2 animals presented LC activation after both doses of ethanol. Interestingly, variances in the sensitivity of LC to acute ethanol treatment have been observed between alcohol-preferring $(\mathrm{P})$ and alcohol-non-preferring $(\mathrm{NP})$ lines, and the Alko-alcohol (AA) and Alko-non-alcohol (ANA) lines, with high-drinking lines exhibiting blunted ethanol-induced c-Fos expression relative to low-drinking lines [31]. Given work linking the LC to the modulation of the aversive properties of ethanol, the reduced activation of the LC observed in the iHDID1 animals may contribute to their reduced sensitivity to the aversive properties of ethanol, and this blunted response may serve as a key mechanism driving binge-like ethanol consumption specifically in the iHDID1 line. However, similar to the A2, since iHDID2 mice also show reduced sensitivity to the aversive effects of ethanol, the link between the LC and sensitivity to the aversive properties of ethanol is not straightforward with respect to the iHDID lines.

Acute ethanol induced significant increases in PBN c-Fos expression across all lines. This finding is in keeping with numerous other studies in rodents utilizing a range of acute ethanol doses [22,32-34]. The precise role of the PBN in ethanol consumption is not fully understood. However, in addition to the role in CTA outlined above, recent work has demonstrated stimulation of a neurotensin expressing CeA-PBN pathway increases ethanol, but not water or quinine intake, and modulates the reinforcing properties of ethanol [35]. This pathway is likely intact in the iHDID1 and iHDID2 animals as, in addition to similar c-Fos expression in the PBN, there were also no difference in ethanol-induced c-Fos expression in the CeA between lines. Given the blunting of A2/LC activation observed in the iHDID1 line, this may lend further support to the hypothesis that pathways involved in processing the rewarding properties of ethanol (CeA-PBN) remain unaltered in the iHDID1 line while pathways mediating the aversive properties (A2/LC-PBN) are impaired.

\subsection{Extended Amygdala Regions}

Given the importance of the CeA in all stages of ethanol abuse and the general assignment of salience to both rewarding and aversive stimuli, it is of little surprise similar patterns of c-Fos activity were observed across all three lines [35-37]. Inhibitory GABAergic cells within the CeA are known to respond to acute ethanol exposure across multiple species and genetic lines, as has been extensively 
reviewed previously [37-39]. Our present findings add to this large body of literature demonstrating an important role of the CeA across the ethanol abuse spectrum.

Also well known to modulate ethanol-directed behaviors and the anxiolytic effects of ethanol, in contrast to the GABAergic CeA, the BLA is composed primarily of glutamatergic pyramidal neurons which are known to be inhibited, rather than activated, by acute ethanol exposure [40]. As these glutamatergic cells display low tonic baseline activation, a decrease in c-Fos levels may be difficult to detect [41]. Thus, the lack of change in c-Fos activity following ethanol exposure in all lines is somewhat expected.

The most notable finding in the areas of the extended amygdala analyzed is the significant increase in BNST c-Fos expression following ethanol exposure in the HS and iHDID2, but not the iHDID1 line. The BNST is known to play a role in modulating neurobiological responses to both appetitive and aversive stimuli [42]. The acute ethanol-induced increase in BNST c-Fos expression observed in the HS and iHDID2 lines is consistent with previous works in Long-Evans and Sprague-Dawley rats and C57BL6/J mice [21,43-46]. The precise impact of impaired ethanol-induced c-Fos expression in the BNST in the iHDID1 line on the drive to consume ethanol is presently unknown. Chronic stress exposure has been shown to attenuate acute ethanol-induced cAMP response element-binding protein (CREB) phosphorylation in the BNST [47]. Further, disruption of glutamatergic signaling within the BNST induces an insensitivity to the aversive properties of ethanol [48]. Together, these results indicate that during acute ethanol exposure, such as used in this work, a primary role of BNST activation may be the processing of the stress-inducing, or aversive, properties of high dose ethanol. The BNST receives numerous glutamatergic and GABAergic afferents, with the CeA known to exert significant control over BNST stress-related signaling (for review see [42,49]). As the CeA response to acute ethanol was not different between lines in this study, the lack of BNST response in the iHDID1 line is likely due to alterations in the BNST itself rather than alterations in CeA signaling to the BNST. This could be due to alterations in signaling from other afferent areas, or changes in receptor expression within the BNST which receive incoming CeA transmission. The CeA is known to modulate BNST activity via both GABAergic and numerous neuropeptide signals, with corticotrophin releasing factor (CRF) and Neuropeptide Y (NPY) being perhaps the most well studied regarding ethanol use disorder research $[37,38,50]$. It would be of future interest to analyze potential alterations in BNST NPY/CRF/GABA receptor expression levels in the iHDID1 line relative to the HS and iHDID2 lines. Notably, NPY expression in the CeA and BNST display significant baseline or ethanol-induced alterations in expression in the HDID lines relative to the HS line, highlighting a potential critical role of this neuropeptide in driving binge-like ethanol consumption in these lines [51]. In contrast, it is important to note lesioning of the BNST or selective pharmacological silencing of BNST projections to the VTA decreases binge-like ethanol intake [52-54]. The precise mechanisms underlying the genetic predisposition towards impaired BNST activation following acute ethanol exposure require further investigation.

\subsection{Midbrain Regions}

No baseline differences or ethanol-induced changes in c-Fos were detected in the LHb. Similar to the above regions, the $\mathrm{LHb}$ has been shown to be critically involved in ethanol-induced CTA, with CTA expression promoting an elevation in tonic $\mathrm{LHb}$ firing and lesion of the LHb sufficient to block CTA development [55]. Indeed, the $\mathrm{LHb}$ plays an overall important role in processing of aversive stimuli and negative reinforcement (for review see [56]). LHb lesion further increases voluntary ethanol consumption and operant responding [57]. Consistent with this, LHb electric stimulation has been found to reduce voluntary ethanol consumption [58]. Interestingly, a lack of change in LHb c-Fos expression following ethanol consumption was found in the $\mathrm{Li}$ and colleagues work, with potent alterations detected only during withdrawal from ethanol exposure [58]. Our findings of no change in $\mathrm{LHb}$ c-Fos expression between the lines following acute ethanol exposure are in keeping with this previous work. Thus, it would be of future interest to observe if $\mathrm{LHb}$ c-Fos expression in the iHDID lines 
is blunted relative to the HS line during withdrawal from ethanol. Interestingly, in Sprague-Dawley rats, low dose acute ethanol injection $(0.25 \mathrm{~g} / \mathrm{kg})$ significantly increased LHb c-Fos in glutamatergic cells, suggesting a potentially dose-dependent role of this area in response to acute exposure which may also be of future interest in evaluation of the iHDID lines [59].

No ethanol-induced changes in $\mathrm{MHb}$ c-Fos were detected in any of the genetic lines; however, the iHDID2 line was found to express significantly higher levels of c-Fos following a saline injection relative to the $\mathrm{HS}$ strain. The $\mathrm{MHb}$ has been recently put forward as a critical area in modulating drug abuse behavior (for review see [60]). The $\mathrm{MHb}$ serves to modulate reward information as a relay station in the transmission of signals from the limbic forebrain to the midbrain and hindbrain [61]. Interestingly, baseline increased glucose utilization rate and a total lack of the inhibitory peptide NPY are found in the $\mathrm{MHb}$ of ethanol-preferring relative to non-preferring rodent genetic lines [62,63]. Further, the NPY system is one of the genes found to show significant changes in connectivity in HDID mice relative to the founder HS stock, and further HDID1 and HDID2 mice display distinct baseline, as well as blunted ethanol-induced alterations in, NPY expression in a region-specific manner [6,51]. This may suggest that $\mathrm{MHb}$ hyperactivity contributes to ethanol-directed behaviors across multiple genetic lines. The precise impact of this hyperactivity has yet to be investigated. Previous work has found mice will voluntarily stimulate the $\mathrm{MHb}$, and that ablation of this region reduces sucrose preference [64]. Interestingly, the $\mathrm{MHb}$ metabolic activity was found to be significantly increased in rats following exposure to chronic unpredictable mild stress and for $\mathrm{MHb}$ lesion to in fact increase sucrose preference in these animals [65]. This suggests either a difference in $\mathrm{MHb}$ role between species, or a potentially state dependent role of the $\mathrm{MHb}$ in mediating reward/anhedonia associated behaviors. This present work offers the first evidence that $\mathrm{MHb}$ baseline activity may contribute to increased ethanol consumption in the iHDID2, but not iHDID1, lines of mice.

The RMTg is another region closely associated with aversive signaling. While no ethanol-induced alterations in RMTg c-Fos were observed in any line, an interesting significant reduction in RMTg activity following saline injection alone was observed in the iHDID1 line relative to the parent HS line. The RMTg serves to regulate ventral tegmental area (VTA) activity via extensive GABAergic innervation (for review see [66]). Critically, this region is thought to participate in limiting voluntary ethanol intake through potent regulation of the aversive properties of ethanol. For example, RMTg lesion results in increased voluntary ethanol consumption and accelerates CTA extinction [67]. Though initially somewhat surprising, the lack of ethanol-induced alteration in c-Fos detected in this work is consistent with previous findings in which only ethanol paired with a CTA environment, but not ethanol administered without this pairing, induced an increase in RMTg c-Fos [68]. It is likely that assessment following voluntary ethanol consumption within an ethanol-paired environment, in contrast to ethanol administered by IP injection in this work, would offer more relevant insight into potential differences in ethanol-induced alterations in RMTg c-Fos expression between the HS and iHDID lines. Interestingly, recent work has suggested that the densely expressed $\mathrm{Mu}$-opioid receptor (MOR) population in the RMTg may play a central role in regulating the balance between the aversive/rewarding properties of ethanol $[69,70]$. This is notable as HDID1 ethanol consumption has been shown to be insensitive to naltrexone (a MOR-antagonist) administration [71]. Taken together, the reduced RMTg activity at baseline detected in this work may suggest that altered MOR regulation of activity in this area serves as a brain region contributing to this naltrexone insensitivity.

\subsection{Other Regions Examined}

PAG activity has previously been shown to be modulated by ethanol exposure. For instance, acute ethanol increases glutamatergic transmission onto PAG dopaminergic neurons in ex vivo recordings, and binge-like ethanol exposure in alcohol-preferring rats alters PAG RNA expression [72,73]. Given the role of the PAG in processing pain, anxiety, and reward processing, and the apparent attenuation of the aversive effects of ethanol in the iHDID lines, we sought to assess any alteration in PAG reactivity to a saline injection or following ethanol exposure relative to the HS line (for review 
see [74]). No differences between lines following saline or ethanol exposure were detected, suggesting a potential lack of PAG involvement in processing of an acute ethanol injection in all lines. This finding is in keeping with previous work which found no change in PAG c-Fos expression following lower dose ethanol exposure in rats [45]. However, given the highly heterogenous composition of the region, it is possible only a subset of PAG cells are engaged by acute ethanol exposure and thus may not be detected by the general assessment of total c-Fos activity. Future work will be required to parse out the specific role of the PAG in the regulation of binge drinking behavior.

The EW likely plays a critical role in modulating ethanol-directed behaviors, as ethanol, but not water, saccharine, or non-alcoholic beer, intake induces increased activity in this region [21,75]. We found acute ethanol exposure increased EW c-Fos expression in the HS and iHDID2 lines, but not the iHDID1 line. Of note, the iHDID1 line expressed significantly higher levels of EW c-Fos expression following a saline injection alone relative to the HS line. This increased activity is unlikely to be due to an increased sensitivity of the iHDID1 line to the stressful stimuli of an IP saline injection, as previous work has shown stress exposure does not induce an increase in EW c-Fos expression [76]. This lack of an ethanol-induced increase in c-Fos expression is therefore potentially due to a ceiling effect of high basal EW activity in the iHDID1 line rather than an insensitivity to ethanol. This region expresses numerous neuromodulators which may contribute to its role in binge-like drinking behavior (for review see [77]. The dense levels of urocortin (Ucn1), a neuropeptide which binds to CRF receptors, expression is the most extensively studied in relation to drug abuse behavior [78]. Indeed, previous studies have found that ethanol induced c-Fos expression in the EW to predominantly colocalize with Ucn1 expression in C57BL/6J mice [79]. Increased levels of Ucn1 expression have been found in numerous rodent lines known for high ethanol preference and intake (for review see [78]). Though Ucn1 levels have not been directly assessed in iHDID1 animals, an increased baseline level of Ucn1 as found in other genetic lines would be in keeping with the higher baseline levels of EW c-Fos activity in the iHDID1 line.

Of regions assessed in this work, the PVT stands as the lone area in which the iHDID2 line differed in ethanol-induced c-Fos expression from the HS founder line. The PVT has been put forward as an integration hub for drug-related hypothalamic signaling [80-82]. The PVT is a primarily glutamatergic structure which sends projections to brain regions known to be critical to ethanol-related behavior, such as the extended amygdala, nucleus accumbens, and prefrontal cortex [82]. Distinct inputs to the PVT have been shown to play a role in reward-related behaviors. Specifically, the orexin system potently regulates PVT signaling and is suggested to primarily act in this region in regulating negative emotional states (for review see [80]; prefrontal cortex glutamatergic inputs have been shown to modulate cue-reward learning [83]; while stimulation of zona incerta GABAergic inputs to the PVT rapidly stimulates binge-food consumption [84]. This role in reward learning/seeking behavior may be of particular interest in ethanol-directed behaviors. Lesion of or kappa-opioid receptor agonism into the PVT prevents reinstatement of ethanol seeking behavior, with this behavior potentially regulated by PVT projections to the nucleus accumbens [85-87]. Interestingly, the PVT is suggested to be involved in the encoding, and not retrieval, of emotionally salient information $[85,88]$. This is of particular note as in this work the lack of ethanol-induced increase in PVT c-Fos in the iHDID2 line is likely due to the significantly higher baseline activation of the PVT observed in this line following saline injection. This suggests that c-Fos levels could not be increased following ethanol exposure due to a ceiling effect. This general heightened activity of the PVT may thus result in a non-discriminatory assignment of salience to stimuli and increased drive to seek consumable substances. While only speculation, such an interpretation would be in keeping with the previously observed non-specific increase in consumption of ethanol, water, and saccharine, as well as the specific increase in bout number, but not bout duration, observed in HDID2 mice [15]. Future studies examining the precise role of the PVT in driving the seeking and consumption of ethanol and other substances in the iHDID2 line would be of particular interest. 


\section{Conclusions}

The results of this work contribute, in combination with previous studies, to characterizing the potential neural mechanisms underlying the drive to binge consume ethanol in two replicate iHDID lines. Our findings lend further support to the previously proposed hypothesis the iHDID1 and the iHDID2 lines arrive at a similar behavior phenotype through divergent genetic mechanisms [6]. Assessing genetic mechanisms underlying resistance to the aversive effects of ethanol may be of particular clinical relevance as sensitivity to aversive ethanol effects such as dizziness and nausea is less severe in sons of alcoholics relative to sons of non-alcoholics, with this constitutional difference proposed to be a factor influencing the difference in risk for alcoholism in these two groups [89]. Given the increasing importance of personalized medicine in treating alcohol use disorder in humans, the iHDID replicate lines stand as an important model in evaluating both potential pharmacotherapies and of the multiple potential mechanisms underlying ethanol-directed behaviors between individuals.

Author Contributions: Conceptualization, N.W.B., M.A.C. and T.E.T.; data curation, S.L.R., A.P.S.D., N.W.B., C.A.H., K.L.L., S.C.B., M.A.C., M.N., and H.W.B.; formal analysis, S.L.R., A.P.S.D., C.A.H., K.L.L., S.C.B. and M.A.C.; funding acquisition, M.A.C., M.N. and T.E.T.; investigation, S.L.R., A.P.S.D. and N.W.B.; methodology, T.E.T.; project administration, R.D.T., M.A.C. and T.E.T.; resources, R.D.T. and T.E.T.; supervision, R.D.T. and T.E.T.; writing-original draft, S.L.R., A.P.S.D., N.W.B., C.A.H., H.W.B., R.D.T., M.A.C. and T.E.T.; writing-review and editing, A.P.S.D. All authors have read and agreed to the published version of the manuscript.

Funding: The authors declare no conflict of interest. This work was supported by National Institutes of Health grants AA022048, AA013573, AA025809, F32AA025811.

Acknowledgments: The authors would like to thank John Crabbe and Pamela Metten for supplying the iHDID1, iHDID2, and HS mice.

Conflicts of Interest: The authors declare no conflict of interest.

\section{References}

1. Thiele, T.E.; Navarro, M. “Drinking in the dark" (DID) procedures: A model of binge-like ethanol drinking in non-dependent mice. Alcohol 2014, 48, 235-241. [CrossRef]

2. Sprow, G.M.; Thiele, T.E. The neurobiology of binge-like ethanol drinking: Evidence from rodent models. Physiol. Behav. 2012, 106, 325-331. [CrossRef]

3. Barkley-Levenson, A.M.; Crabbe, J.C. High Drinking in the Dark Mice: A genetic model of drinking to intoxication. Alcohol 2014, 48, 217-223. [CrossRef]

4. Crabbe, J.C.; Metten, P.; Savarese, A.M.; Ozburn, A.R.; Schlumbohm, J.P.; Spence, S.E.; Hack, W.R. Ethanol conditioned taste aversion in high drinking in the dark mice. Brain Sci. 2019, 9, 2. [CrossRef] [PubMed]

5. Barkley-Levenson, A.M.; Crabbe, J.C. Distinct ethanol drinking microstructures in two replicate lines of mice selected for drinking to intoxication. Genes Brain Behav. 2015, 14, 398-410. [CrossRef]

6. Dan Iancu, O.; Oberbeck, D.; Darakjian, P.; Metten, P.; Mcweeney, S.; Crabbe, J.C.; Hitzemann, R. Selection for Drinking in the Dark Alters Brain Gene Coexpression Networks. Alcohol. Clin. Exp. Res. 2013, 37, 1295-1303. [CrossRef]

7. Grant, K.A.; Leng, X.; Green, H.L.; Szeliga, K.T.; Rogers, L.S.; Gonzales, S.W. Drinking typography established by scheduled induction predicts chronic heavy drinking in a monkey model of ethanol self-administration. Alcohol. Clin. Exp. Res. 2008, 32, 1824-1838. [CrossRef]

8. Berridge, K.C. Measuring hedonic impact in animals and infants: Microstructure of affective taste reactivity patterns. Neurosci. Biobehav. Rev. 2000, 24, 173-198. [CrossRef]

9. Robinson, S.L.; McCool, B.A. Microstructural analysis of rat ethanol and water drinking patterns using a modified operant self-administration model. Physiol. Behav. 2015, 149, 119-130. [CrossRef]

10. Barkley-Levenson, A.M.; Cunningham, C.L.; Smitasin, P.J.; Crabbe, J.C. Rewarding and aversive effects of ethanol in High Drinking in the Dark selectively bred mice. Addict. Biol. 2015, 20, 80-90. [CrossRef]

11. Barkley-Levenson, A.M.; Crabbe, J.C. Genotypic and sex differences in anxiety-like behavior and alcohol-induced anxiolysis in High Drinking in the Dark selected mice. Alcohol 2015, 49, $29-36$. [CrossRef] [PubMed] 
12. Parker, C.C.; Lusk, R.; Saba, L.M. Alcohol Sensitivity as an Endophenotype of Alcohol Use Disorder: Exploring Its Translational Utility between Rodents and Humans. Brain Sci. 2020, 10, 725. [CrossRef] [PubMed]

13. Franklin, K.; Paxinos, G. The Mouse Brain in Stereotaxic Coordinates, Compact, 3rd ed.; Academic Press Inc.: Cambridge, MA, USA, 2008; ISBN 9780123742445.

14. De Chaumont, F.; Dallongeville, S.; Chenouard, N.; Hervé, N.; Pop, S.; Provoost, T.; Meas-Yedid, V.; Pankajakshan, P.; Lecomte, T.; le Montagner, Y.; et al. Icy: An open bioimage informatics platform for extended reproducible research. Nat. Methods 2012, 9, 690-696. [CrossRef]

15. Barkley-Levenson, A.M.; Crabbe, J.C. Ethanol drinking microstructure of a high drinking in the dark selected mouse line. Alcohol. Clin. Exp. Res. 2012, 36, 1330-1339. [CrossRef]

16. Crabbe, J.C.; Spence, S.E.; Brown, L.L.; Metten, P. Alcohol preference drinking in a mouse line selectively bred for high drinking in the dark. Alcohol 2011, 45, 427-440. [CrossRef]

17. Bernstein, I.L.; Koh, M.T. Molecular signaling during taste aversion learning. Chem. Senses 2007, 32, 99-103. [CrossRef]

18. Palmiter, R.D. The Parabrachial Nucleus: CGRP Neurons Function as a General Alarm. Trends Neurosci. 2018, 41, 280-293. [CrossRef]

19. Smith, R.J.; Aston-Jones, G. Noradrenergic transmission in the extended amygdala: Role in increased drug-seeking and relapse during protracted drug abstinence. Brain Struct. Funct. 2008, 213, 43-61. [CrossRef]

20. Aimino, M.A.; Coker, C.R.; Silberman, Y. Acute ethanol modulation of neurocircuit function in the nucleus of the tractus solitarius. Brain Res. Bull. 2018, 138, 5-11. [CrossRef]

21. Burnham, N.W.; Thiele, T.E. Voluntary Binge-like Ethanol Consumption Site-specifically Increases c-Fos Immunoexpression in Male C57BL6/J Mice. Neuroscience 2017, 367, 159-168. [CrossRef]

22. Thiele, T.E.; Roitman, M.F.; Bernstein, I.L. c-Fos induction in rat brainstem in response to ethanol- and lithium chloride-induced conditioned taste aversions. Alcohol. Clin. Exp. Res. 1996, 20, 1023-1028. [CrossRef]

23. Lindvall, O.; Bjorklund, A. The organization of the ascending catecholamine neuron systems in the rat brain. As revealed by the glyoxylic acid fluorescence method. Acta Physiol. Scand. 1974, 92, 1-48.

24. Aston-Jones, G.; Waterhouse, B. Locus coeruleus: From global projection system to adaptive regulation of behavior. Brain Res. 2016, 1645, 75-78. [CrossRef]

25. Benarroch, E.E. Locus coeruleus. Cell Tissue Res. 2018, 373, 221-232. [CrossRef]

26. Deal, A.L.; Mikhailova, M.A.; Grinevich, V.P.; Weiner, J.L.; Gainetdinov, R.R.; Budygin, E.A. In vivo voltammetric evidence that locus coeruleus activation predominantly releases norepinephrine in the infralimbic cortex: Effect of acute ethanol. Synapse 2019, 73, e22080. [CrossRef]

27. Vilpoux, C.; Warnault, V.; Pierrefiche, O.; Daoust, M.; Naassila, M. Ethanol-sensitive brain regions in rat and mouse: A cartographic review, using immediate early gene expression. Alcohol. Clin. Exp. Res. 2009, 33, 945-969. [CrossRef]

28. Thiele, T.E.; Cubero, I.; van Dijk, G.; Mediavilla, C.; Bernstein, I.L. Ethanol-Induced c-Fos Expression in Catecholamine- and Neuropeptide Y-Producing Neurons in Rat Brainstem. Alcohol. Clin. Exp. Res. 2000, 24, 802-809. [CrossRef]

29. Hirschberg, S.; Li, Y.; Randall, A.; Kremer, E.J.; Pickering, A.E. Functional dichotomy in spinal- vs prefrontal-projecting locus coeruleus modules splits descending noradrenergic analgesia from ascending aversion and anxiety in rats. eLife 2017, 6, e29808. [CrossRef]

30. Bonaz, B.; Taché, Y. Water-avoidance stress-induced c-fos expression in the rat brain and stimulation of fecal output: Role of corticotropin-releasing factor. Brain Res. 1994, 641, 21-28. [CrossRef]

31. Thiele, T.E.; van Dijk, G.; Bernstein, I.L. Ethanol-induced c-Fos expression in rat lines selected for low and high alcohol consumption. Brain Res. 1997, 756, 278-282. [CrossRef]

32. Canales, J.J. Catalase-independent early-gene expression in rat brain following acute ethanol exposure. Brain Res. 2004, 1016, 96-101. [CrossRef]

33. Chang, S.L.; Patel, N.A.; Romero, A.A. Activation and desensitization of Fos immunoreactivity in the rat brain following ethanol administration. Brain Res. 1995, 679, 89-98. [CrossRef]

34. Kolodziejska-Akiyama, K.M.; Young, M.C.; Jiang, Y.; Loh, H.H.; Chang, S.L. Ethanol-induced FOS immunoreactivity in the brain of $\mu$-opioid receptor knockout mice. Drug Alcohol Depend. 2005, 80, 161-168. [CrossRef]

35. Torruella-Suárez, M.L.; Vandenberg, J.R.; Cogan, E.S.; Tipton, G.J.; Teklezghi, A.; Dange, K.; Patel, G.K.; McHenry, J.A.; Andrew Hardaway, J.; Kantak, P.A.; et al. Manipulations of central amygdala neurotensin neurons alter the consumption of ethanol and sweet fluids in mice. J. Neurosci. 2020, 40, 632-647. [CrossRef] 
36. Kim, J.; Zhang, X.; Muralidhar, S.; LeBlanc, S.A.; Tonegawa, S. Basolateral to Central Amygdala Neural Circuits for Appetitive Behaviors. Neuron 2017, 93, 1464-1479. [CrossRef]

37. Gilpin, N.W.; Herman, M.A.; Roberto, M. The central amygdala as an integrative hub for anxiety and alcohol use disorders. Biol. Psychiatry 2015, 77, 859-869. [CrossRef]

38. Roberto, M.; Gilpin, N.W.; Siggins, G.R. The central amygdala and alcohol: Role of $\gamma$-aminobutyric acid, glutamate, and neuropeptides. Cold Spring Harb. Perspect. Med. 2012, 2, a012195. [CrossRef]

39. Roberto, M.; Madamba, S.G.; Stouffer, D.G.; Parsons, L.H.; Siggins, G.R. Increased GABA release in the central amygdala of ethanol-dependent rats. J. Neurosci. 2004, 24, 10159-10166. [CrossRef]

40. McCool, B.A.; Christian, D.T.; Diaz, M.R.; Läck, A.K. Glutamate plasticity in the drunken amygdala: The making of an anxious synapse. Int. Rev. Neurobiol. 2010, 91, 205-233. [CrossRef]

41. Rainnie, D.G.; Asprodini, E.K.; Shinnick-Gallagher, P. Excitatory transmission in the basolateral amygdala. J. Neurophysiol. 1991, 66, 986-998. [CrossRef]

42. Ch'ng, S.; Fu, J.; Brown, R.M.; McDougall, S.J.; Lawrence, A.J. The intersection of stress and reward: BNST modulation of aversive and appetitive states. Prog. Neuro-Psychopharmacol. Biol. Psychiatry 2018, 87, 108-125. [CrossRef] [PubMed]

43. Sharko, A.C.; Kaigler, K.F.; Fadel, J.R.; Wilson, M.A. Ethanol-induced anxiolysis and neuronal activation in the amygdala and bed nucleus of the stria terminalis. Alcohol 2016, 50, 19-25. [CrossRef] [PubMed]

44. Saalfield, J.; Spear, L. Fos activation patterns related to acute ethanol and conditioned taste aversion in adolescent and adult rats. Alcohol 2019, 78, 57-68. [CrossRef] [PubMed]

45. Ryabinin, A.E.; Criado, J.R.; Henriksen, S.J.; Bloom, F.E.; Wilson, M.C. Differential sensitivity of c-Fos expression in hippocampus and other brain regions to moderate and low doses of alcohol. Mol. Psychiatry 1997, 2, 32-43. [CrossRef]

46. Crankshaw, D.L.; Briggs, J.E.; Olszewski, P.K.; Shi, Q.; Grace, M.K.; Billington, C.J.; Levine, A.S. Effects of intracerebroventricular ethanol on ingestive behavior and induction of c-Fos immunoreactivity in selected brain regions. Physiol. Behav. 2003, 79, 113-120. [CrossRef]

47. Schreiber, A.L.; McGinn, M.A.; Edwards, S.; Gilpin, N.W. Predator odor stress blunts alcohol conditioned aversion. Neuropharmacology 2019, 144, 82-90. [CrossRef]

48. Campbell, R.R.; Domingo, R.D.; Williams, A.R.; Wroten, M.G.; McGregor, H.A.; Waltermire, R.S.; Greentree, D.I.; Goulding, S.P.; Thompson, A.B.; Lee, K.M.; et al. Increased alcohol-drinking induced by manipulations of mGlu5 phosphorylation within the bed nucleus of the stria terminalis. J. Neurosci. 2019, 39, 2745-2761. [CrossRef]

49. Centanni, S.W.; Bedse, G.; Patel, S.; Winder, D.G. Driving the Downward Spiral: Alcohol-Induced Dysregulation of Extended Amygdala Circuits and Negative Affect HHS Public Access. Alcohol. Clin. Exp. Res. 2019, 43, 2000-2013. [CrossRef]

50. Robinson, S.L.; Thiele, T.E. The Role of Neuropeptide Y (NPY) in Alcohol and Drug Abuse Disorders. Int. Rev. Neurobiol. 2017, 136, 177-197. [CrossRef]

51. Barkley-Levenson, A.M.; Ryabinin, A.E.; Crabbe, J.C. Neuropeptide Y response to alcohol is altered in nucleus accumbens of mice selectively bred for drinking to intoxication. Behav. Brain Res. 2016, 302, 160-170. [CrossRef]

52. Dhaher, R.; Finn, D.; Snelling, C.; Hitzemann, R. Lesions of the extended amygdala in C57BL/6J mice do not block the intermittent ethanol vapor-induced increase in ethanol consumption. Alcohol. Clin. Exp. Res. 2008, 32, 197-208. [CrossRef] [PubMed]

53. Rinker, J.A.; Marshall, S.A.; Mazzone, C.M.; Lowery-Gionta, E.G.; Gulati, V.; Pleil, K.E.; Kash, T.L.; Navarro, M.; Thiele, T.E. Extended Amygdala to Ventral Tegmental Area Corticotropin-Releasing Factor Circuit Controls Binge Ethanol Intake. Biol. Psychiatry 2017, 81, 930-940. [CrossRef] [PubMed]

54. Companion, M.A.; Thiele, T.E. Assessment of ventral tegmental area-projecting GABAergic neurons from the bed nucleus of the stria terminalis in modulating binge-like ethanol intake. Eur. J. Neurosci. 2018, 48, 3335-3343. [CrossRef] [PubMed]

55. Tandon, S.; Keefe, K.A.; Taha, S.A. Excitation of lateral habenula neurons as a neural mechanism underlying ethanol-induced conditioned taste aversion. J. Physiol. 2017, 595, 1393-1412. [CrossRef] [PubMed]

56. Shah, A.; Zuo, W.; Kang, S.; Li, J.; Fu, R.; Zhang, H.; Bekker, A.; Ye, J.H. The lateral habenula and alcohol: Role of glutamate and M-type potassium channels. Pharmacol. Biochem. Behav. 2017, 162, 94-102. [CrossRef] 
57. Haack, A.K.; Sheth, C.; Schwager, A.L.; Sinclair, M.S.; Tandon, S.; Taha, S.A. Lesions of the Lateral Habenula Increase Voluntary Ethanol Consumption and Operant Self-Administration, Block Yohimbine-Induced Reinstatement of Ethanol Seeking, and Attenuate Ethanol-Induced Conditioned Taste Aversion. PLoS ONE 2014, 9, e92701. [CrossRef]

58. Li, J.; Zuo, W.; Fu, R.; Xie, G.; Kaur, A.; Bekker, A.; Hong Ye, J.; Ye, J.-H. High Frequency Electrical Stimulation of Lateral Habenula Reduces Voluntary Ethanol Consumption in Rats. Int. J. Neuropsychopharmacol. 2016, 19, pyw050. [CrossRef]

59. Fu, R.; Mei, Q.; Zuo, W.; Li, J.; Gregor, D.; Bekker, A.; Ye, J. Low-dose ethanol excites lateral habenula neurons projecting to VTA, RMTg, and raphe. Int. J. Physiol. Pathophysiol. Pharmacol. 2017, 9, 217-230.

60. McLaughlin, I.; Dani, J.A.; de Biasi, M. The medial habenula and interpeduncular nucleus circuitry is critical in addiction, anxiety, and mood regulation. J. Neurochem. 2017, 142, 130-143. [CrossRef]

61. Sutherland, R.J. The dorsal diencephalic conduction system: A review of the anatomy and functions of the habenular complex. Neurosci. Biobehav. Rev. 1982, 6, 1-13. [CrossRef]

62. Hwang, B.H.; Suzuki, R.; Lumeng, L.; Li, T.K.; McBride, W.J. Innate differences in neuropeptide Y (NPY) mRNA expression in discrete brain regions between alcohol-preferring $(\mathrm{P})$ and -nonpreferring $(\mathrm{NP})$ rats: A significantly low level of NPY mRNA in dentate gyrus of the hippocampus and absence of NPY mRNA in the medial habenular nucleus of P rats. Neuropeptides 2004, 38, 359-368. [CrossRef] [PubMed]

63. Smith, D.G.; Learn, J.E.; McBride, W.J.; Lumeng, L.; Li, T.K.; Murphy, J.M. Alcohol-naïve alcohol-preferring (P) rats exhibit higher local cerebral glucose utilization than alcohol-nonpreferring (NP) and wistar rats. Alcohol. Clin. Exp. Res. 2001, 25, 1309-1316. [CrossRef] [PubMed]

64. Hsu, Y.W.A.; Wang, S.D.; Wang, S.; Morton, G.; Zariwala, H.A.; de la Iglesia, H.O.; Turner, E.E. Role of the dorsal medial habenula in the regulation of voluntary activity, motor function, hedonic state, and primary reinforcement. J. Neurosci. 2014, 34, 11366-11384. [CrossRef] [PubMed]

65. Xu, C.; Sun, Y.; Cai, X.; You, T.; Zhao, H.; Li, Y.; Zhao, H. Medial habenula-interpeduncular nucleus circuit contributes to anhedonia-like behavior in a rat model of depression. Front. Behav. Neurosci. 2018, 12, 238. [CrossRef] [PubMed]

66. Bourdy, R.; Barrot, M. A new control center for dopaminergic systems: Pulling the VTA by the tail. Trends Neurosci. 2012, 35, 681-690. [CrossRef]

67. Sheth, C.; Furlong, T.M.; Keefe, K.A.; Taha, S.A. Lesion of the rostromedial tegmental nucleus increases voluntary ethanol consumption and accelerates extinction of ethanol-induced conditioned taste aversion. Psychopharmacology 2016, 233, 3737-3749. [CrossRef]

68. Glover, E.J.; McDougle, M.J.; Siegel, G.S.; Jhou, T.C.; Chandler, L.J. Role for the Rostromedial Tegmental Nucleus in Signaling the Aversive Properties of Alcohol. Alcohol. Clin. Exp. Res. 2016, 40, 1651-1661. [CrossRef]

69. Fu, R.; Chen, X.; Zuo, W.; Li, J.; Kang, S.; Zhou, L.H.; Siegel, A.; Bekker, A.; Ye, J.H. Ablation of $\mu$ opioid receptor-expressing GABA neurons in rostromedial tegmental nucleus increases ethanol consumption and regulates ethanol-related behaviors. Neuropharmacology 2016, 107, 58-67. [CrossRef]

70. Ye, J.H.; Fu, R.; He, W. The rostromedial tegmental nucleus and alcohol addiction. Oncotarget 2017, 8, 18624-18625. [CrossRef]

71. Crabbe, J.C.; Ozburn, A.R.; Metten, P.; Barkley-Levenson, A.; Schlumbohm, J.P.; Spence, S.E.; Hack, W.R.; Huang, L.C. High Drinking in the Dark (HDID) mice are sensitive to the effects of some clinically relevant drugs to reduce binge-like drinking. Pharmacol. Biochem. Behav. 2017, 160, 55-62. [CrossRef]

72. Li, C.; McCall, N.M.; Lopez, A.J.; Kash, T.L. Alcohol effects on synaptic transmission in periaqueductal gray dopamine neurons. Alcohol 2013, 47, 279-287. [CrossRef] [PubMed]

73. Mcclintick, J.N.; Mcbride, W.J.; Bell, R.L.; Ding, Z.M.; Liu, Y.; Xuei, X.; Edenberg, H.J. Gene Expression Changes in Glutamate and GABA-A Receptors, Neuropeptides, Ion Channels, and Cholesterol Synthesis in the Periaqueductal Gray Following Binge-Like Alcohol Drinking by Adolescent Alcohol-Preferring (P) Rats. Alcohol. Clin. Exp. Res. 2016, 40, 955-968. [CrossRef]

74. Motta, S.C.; Carobrez, A.P.; Canteras, N.S. The periaqueductal gray and primal emotional processing critical to influence complex defensive responses, fear learning and reward seeking. Neurosci. Biobehav. Rev. 2017, 76, 39-47. [CrossRef] [PubMed]

75. Topple, A.N.; Hunt, G.E.; McGregor, I.S. Possible neural substrates of beer-craving in rats. Neurosci. Lett. 1998, 252, 99-102. [CrossRef] 
76. Turek, V.F.; Ryabinin, A.E. Expression of c-Fos in the mouse Edinger-Westphal nucleus following ethanol administration is not secondary to hypothermia or stress. Brain Res. 2005, 1063, 132-139. [CrossRef]

77. Zuniga, A.; Ryabinin, A.E. Involvement of centrally projecting edinger-westphal nucleus neuropeptides in actions of addictive drugs. Brain Sci. 2020, 10, 67. [CrossRef]

78. Ryabinin, A.E.; Weitemier, A.Z. The urocortin 1 neurocircuit: Ethanol-sensitivity and potential involvement in alcohol consumption. Brain Res. Rev. 2006, 52, 368-380. [CrossRef]

79. Ryabinin, A.E.; Galvan-Rosas, A.; Bachtell, R.K.; Risinger, F.O. High alcohol/sucrose consumption during dark circadian phase in $\mathrm{C} 57 \mathrm{Bl} / 6 \mathrm{~J}$ mice: Involvement of hippocampus, lateral septum and urocortin-positive cells of the Edinger-Westphal nucleus. Psychopharmacology 2003, 165, 296-305. [CrossRef]

80. Martin-Fardon, R.; Boutrel, B. Orexin/hypocretin (Orx/Hcrt) transmission and drug-seeking behavior: Is the paraventricular nucleus of the thalamus (PVT) part of the drug seeking circuitry? Front. Behav. Neurosci. 2012, 6, 75. [CrossRef]

81. Matzeu, A.; Zamora-Martinez, E.R.; Martin-Fardon, R. The paraventricular nucleus of the thalamus is recruited by both natural rewards and drugs of abuse: Recent evidence of a pivotal role for orexin/hypocretin signaling in this thalamic nucleus in drug-seeking behavior. Front. Behav. Neurosci. 2014, 8, 117. [CrossRef]

82. James, M.H.; Dayas, C.V. What about me ... ? The PVT: A role for the paraventricular thalamus (PVT) in drug-seeking behavior. Front. Behav. Neurosci. 2013, 7, 18. [CrossRef] [PubMed]

83. Otis, J.M.; Namboodiri, V.M.K.; Matan, A.M.; Voets, E.S.; Mohorn, E.P.; Kosyk, O.; McHenry, J.A.; Robinson, J.E.; Resendez, S.L.; Rossi, M.A.; et al. Prefrontal cortex output circuits guide reward seeking through divergent cue encoding. Nature 2017, 543, 103-107. [CrossRef] [PubMed]

84. Zhang, X.; van den Pol, A.N. Rapid binge-like eating and body weight gain driven by zona incerta GABA neuron activation. Science 2017, 356, 853-859. [CrossRef]

85. Otis, J.M.; Zhu, M.H.; Namboodiri, V.M.K.; Cook, C.A.; Kosyk, O.; Matan, A.M.; Ying, R.; Hashikawa, Y.; Hashikawa, K.; Trujillo-Pisanty, I.; et al. Paraventricular Thalamus Projection Neurons Integrate Cortical and Hypothalamic Signals for Cue-Reward Processing. Neuron 2019, 103, 423-431. [CrossRef] [PubMed]

86. Marchant, N.J.; Furlong, T.M.; McNally, G.P. Medial dorsal hypothalamus mediates the inhibition of reward seeking after extinction. J. Neurosci. 2010, 30, 14102-14115. [CrossRef] [PubMed]

87. Hamlin, A.S.; Clemens, K.J.; Choi, E.A.; McNally, G.P. Paraventricular thalamus mediates context-induced reinstatement (renewal) of extinguished reward seeking. Eur. J. Neurosci. 2009, 29, 802-812. [CrossRef] [PubMed]

88. Do-Monte, F.H.; Quinõnes-Laracuente, K.; Quirk, G.J. A temporal shift in the circuits mediating retrieval of fear memory. Nature 2015, 519, 460-463. [CrossRef] [PubMed]

89. Schuckit, M.A. Biological markers in alcoholism. Prog. Neuropsychopharmacol. Biol. Psychiatry 1986, 10, 191-199. [CrossRef]

Publisher's Note: MDPI stays neutral with regard to jurisdictional claims in published maps and institutional affiliations.

(C) 2020 by the authors. Licensee MDPI, Basel, Switzerland. This article is an open access article distributed under the terms and conditions of the Creative Commons Attribution (CC BY) license (http://creativecommons.org/licenses/by/4.0/). 